\title{
Resonant averaging for small-amplitude solutions of stochastic NLS equations
}

\author{
Sergei Kuksin, Alberto Maiocchi §
}

\begin{abstract}
We consider the free linear Schrödinger equation on a torus $\mathbb{T}^{d}$, perturbed by a hamiltonian nonlinearity, driven by a random force and damped by a linear damping:

$$
u_{t}-i \Delta u+i \nu \rho|u|^{2 q_{*}} u=-\nu f(-\Delta) u+\sqrt{\nu} \frac{d}{d t} \sum_{\mathbf{k} \in \mathbb{Z}^{d}} b_{\mathbf{k}} \boldsymbol{\beta}^{\mathbf{k}}(t) e^{i \mathbf{k} \cdot x}
$$

Here $u=u(t, x), x \in \mathbb{T}^{d}, 0<\nu \ll 1, q_{*} \in \mathbb{N}, f$ is a positive continuous function, $\rho$ is a positive parameter and $\boldsymbol{\beta}^{\mathbf{k}}(t)$ are standard independent complex Wiener processes. We are interested in limiting, as $\nu \rightarrow 0$, behaviour of distributions of solutions for this equation and of its stationary measure. Writing the equation in the slow time $\tau=\nu t$, we prove that the limiting behaviour of the both is described by the effective equation

$$
u_{\tau}+f(-\Delta) u=-i F(u)+\frac{d}{d \tau} \sum b_{\mathbf{k}} \boldsymbol{\beta}^{\mathbf{k}}(\tau) e^{i \mathbf{k} \cdot x},
$$

where the nonlinearity $F(u)$ is made out of the resonant terms of the monomial $|u|^{2 q_{*}} u$.
\end{abstract}

\section{Contents}

$\mathbf{0}$ Introduction $\quad 2$

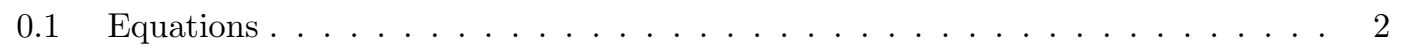

0.2 Discrete Turbulence . . . . . . . . . . . . . . . . . . . . . 3

0.3 Inviscid limits for damped/driven hamiltonian PDE, effective equations and

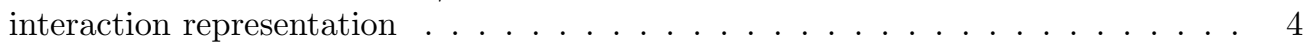

0.4 Results ............................ 7

0.5 Weak Turbulence .......................... 9

1 Preliminaries 10

1.1 Apriori estimates. . . . . . . . . . . . . . . . . . . . . . . 10

1.2 Resonant averaging ......................... . . . . . . . . . . . . . . . . . 13

1.3 Resonant averaging in a Hilbert space . . . . . . . . . . . . . . . . . 13

${ }^{*}$ CNRS and I.M.J, Université Paris Diderot-Paris 7, Paris, France, e-mail: kuksin@math.jussieu.fr

$\S$ Laboratoire de Mathématiques, Université de Cergy-Pontoise, 2 avenue Adolphe Chauvin, Cergy-Pontoise, France, e-mail: alberto.maiocchi@unimi.it 
2 Averaging for equation (1.2).

2.1 Equation (1.2) in $v$-variables, resonant monomials and combinations of phases. . 14

2.2 Averaged equations, effective equation, interaction representation . . . . . . . . 18

2.3 Properties of resonant Hamiltonian $\mathcal{H}^{\text {res }}$ and effective equation . . . . . . . . . . 21

\section{Explicit calculation}

4.1 Averaging theorem for the initial-value problem. . . . . . . . . . . . . . . 24

4.2 Averaging theorem for stationary solutions. . . . . . . . . . . . . . . . 27

4.3 Mixing in the effective equations . . . . . . . . . . . . . . 30

4.4 Proof of Lemma $4.6 \ldots \ldots \ldots$. . . . . . . . . . . . . . . . . . . . . . . . . . . .

\section{A Proof of Proposition 4.10}

\section{Introduction}

\section{$0.1 \quad$ Equations}

The nonlinear Schrödinger equation on the torus with small nonlinearity

$$
u_{t}(t, x)-i \Delta u(t, x)=-i \varepsilon^{2 q_{*}}|u|^{2 q_{*}} u, \quad u=u(t, x), \quad x \in \mathbb{T}_{L}^{d}=\mathbb{R}^{d} /\left(2 \pi L \mathbb{Z}^{d}\right),
$$

where $q_{*} \in \mathbb{N}$ and $0<\varepsilon \leq 1$, is a popular model in various branches of science. The nonlinearity in (0.3) is hamiltonian and may be written as

$$
-i \varepsilon^{2 q_{*}}|u|^{2 q_{*}} u=\varepsilon^{2 q_{*}} i \nabla \mathcal{H}(u), \quad \mathcal{H}(u)=\mathcal{H}^{2 q_{*}+2}(u)=-\frac{1}{2 q_{*}+2} \int|u(x)|^{2 q_{*}+2} d x,
$$

so the equation describes a conservative system. To describe systems, interacting with the "environment", physicists often add at some stage (to this and to other similar equations) terms, describing pumping the energy to the system and its dissipation (e.g. see [ZLF92], Section 2.2.3). A way to describe the pumping of energy is by adding to the equation a small random force, which usually is Gaussian, smooth in $x$, and often is white in time $t$, while to describe the dissipation a suitable function of the Laplacian usually is used. In this way we arrive at the equation (cf. [ZL75], eq. (5), and [CFG08], Section 1.2, eq. (1.2))

$$
u_{t}-i \Delta u=-i \varepsilon^{2 q_{*}}|u|^{2 q_{*}} u-\nu f(-\Delta) u+\sqrt{\nu} \frac{d}{d t} \sum_{\mathbf{k} \in \mathbb{Z}_{L}^{d}} b_{\mathbf{k}} \boldsymbol{\beta}^{\mathbf{k}}(t) e^{i \mathbf{k} \cdot x} . \quad x \in \mathbb{T}_{L}^{d},
$$

Here $0<\nu \leq 1$ and $\mathbb{Z}_{L}^{d}$ denotes the set of vectors of the form $\mathbf{k}=\mathbf{l} / L$ with $\mathbf{l} \in \mathbb{Z}^{d}$. The damping $-f(-\Delta)$ is the selfadjoint linear operator in $L_{2}\left(\mathbb{T}_{L}^{d}\right)$ which acts on the exponents $e^{i \mathbf{k} \cdot x}, \mathbf{k} \in \mathbb{Z}_{L}^{d}$, according to

$$
f(-\Delta) e^{i \mathbf{k} \cdot x}=\gamma_{\mathbf{k}} e^{i \mathbf{k} \cdot x}, \quad \gamma_{\mathbf{k}}=f\left(\lambda_{\mathbf{k}}\right) \quad \text { where } \quad \lambda_{\mathbf{k}}=|\mathbf{k}|^{2} .
$$

The real-valued smooth function $f(t), t \geq 0$, is positive and $f^{\prime}>0$. To avoid technicalities, not relevant for this work, we assume that $f(t) \geq C_{1}|t|+C_{2}$ for all $t$, for suitable positive 
constants $C_{1}, C_{2}$ (for example, $f(-\Delta) u=-\Delta u+u$ ). The processes $\boldsymbol{\beta}^{\mathbf{k}}, \mathbf{k} \in \mathbb{Z}_{L}^{d}$, are standard independent complex Wiener processes, i.e., $\boldsymbol{\beta}^{\mathbf{k}}(t)=\beta_{+}^{\mathbf{k}}(t)+i \beta_{-}^{\mathbf{k}}(t)$, where $\beta_{ \pm}^{\mathbf{k}}(t)$ are standard independent real Wiener processes. The real numbers $b_{\mathbf{k}}$ are all non-zero and decay fast when $|\mathbf{k}| \rightarrow \infty$. The factor in front of the random force is chosen to be $\sqrt{\nu}$ to guarantee that solutions of $(0.3)$ stay of order one when $t \gg 1$ and $0<\nu \ll 1$.

We assume that eq. (0.3) with sufficiently smooth initial data $u(0, x)=u_{0}(x)$ is well posed. It is well known that this assumption holds (at least) under some restriction on $d, q_{*}$ and the growth of $f(t)$ at infinity, see in Section 1.1

The parameters $\nu$ and $\varepsilon$ measure, respectively, the inverse time-scale of the forced oscillations, and their amplitude. Physicists consider different regimes, where the two parameters are tied in various ways. ${ }^{1}$ To do this they assume some relations between $\varepsilon$ and $\nu$, explicitly or implicitly. In our work we choose

$$
\varepsilon^{2 q_{*}}=\rho \nu,
$$

where $\rho>0$ is a constant. This assumption is within the usually imposed bounds, see [Naz11]. Passing to the slow time $\tau=\nu t$, we get the rescaled equation

$$
\dot{u}+i \nu^{-1}(-\Delta u)=-f(-\Delta) u-i \rho|u|^{2 q_{*}} u+\sum b_{\mathbf{k}} \dot{\boldsymbol{\beta}}^{\mathbf{k}}(\tau) e^{i \mathbf{k} \cdot x},
$$

where $u=u(\tau, x), x \in \mathbb{T}_{L}^{d}$ and the upper dot ${ }^{\cdot}$ stands for $\frac{d}{d \tau}$. If we write $u(\tau, x)$ as Fourier series, $u(\tau, x)=\sum_{\mathbf{k}} v_{\mathbf{k}}(\tau) e^{i \mathbf{k} \cdot x}$, then in view of (0.2), eq. (0.5) may be written as the system

$$
\dot{v}_{\mathbf{k}}+i \nu^{-1} \lambda_{\mathbf{k}} v_{\mathbf{k}}=-\gamma_{\mathbf{k}} v_{\mathbf{k}}+2 \rho i \frac{\partial \mathcal{H}(v)}{\partial \bar{v}_{\mathbf{k}}}+b_{\mathbf{k}} \dot{\beta}^{\mathbf{k}}(\tau), \quad \mathbf{k} \in \mathbb{Z}_{L}^{d} .
$$

Here $\mathcal{H}(v)$ is the Hamiltonian $\mathcal{H}$, expressed in terms of the Fourier coefficients $v=\left(v_{\mathbf{k}}, \mathbf{k} \in \mathbb{Z}_{L}^{d}\right)$ :

$$
\mathcal{H}(v)=-\frac{1}{2 q_{*}+2} \sum_{\mathbf{k}_{1}, \ldots \mathbf{k}_{2 q_{*}+2} \in \mathbb{Z}_{L}^{d}} v_{\mathbf{k}_{1}} \ldots v_{\mathbf{k}_{q_{*}+1}} \bar{v}_{\mathbf{k}_{q_{*}+2}} \ldots \bar{v}_{\mathbf{k}_{2 q_{*}+2}} \delta_{q_{*}+2 \ldots 2 q_{*}+2}^{1 \ldots q_{*}+1},
$$

and we use the standard notation (see [Naz11]):

$$
\delta_{q_{*}+2 \ldots 2 q_{*}+2}^{1 \ldots q_{*}+1}=\left\{\begin{array}{cc}
1 & \text { if } \mathbf{k}_{1}+\ldots+\mathbf{k}_{q_{*}+1}-\mathbf{k}_{q_{*}+2}-\ldots-\mathbf{k}_{2 q_{*}+2}=0 \\
0 & \text { otherwise }
\end{array} .\right.
$$

As before we are interested in the limit $\nu \rightarrow 0 .^{2}$

We note that the method of our work applies as well to equations (0.6) with the Hamiltonians $\mathcal{H}$ of the form (0.2), where the density of the Hamiltonian is a real-valued polynomial of $u$ and $\bar{u}$ (not necessarily a polynomial of $|u|^{2}$ ). For instance, we could work with the cubic Hamiltonians $\mathcal{H}^{3}=\int|u|^{2}(u+\bar{u}) d x$ or $\mathcal{H}^{3}=\int\left(u^{3}+\bar{u}^{3}\right) d x$.

\subsection{Discrete Turbulence}

In physics equations $(0.1)$ and $(0.5)$ with $\nu, \varepsilon \ll 1$ are treated by the theory of weak turbulence (WT); see the works, quoted above. That theory either deals with equation (0.5), where $L=\infty$

\footnotetext{
${ }^{1}$ When forcing and dissipation are not present, a parameter $T$ is introduced, which measures the time scale on which averaging is performed (see [Naz11]): to heuristically compare with the present case, we put $\nu=1 / T$.

${ }^{2}$ See [KN13] for a theory of equation (0.5) for the case when $f(t)=t+1$ and $\nu=\infty$.
} 
by formal replacing Fourier series for $L$-periodic functions with Fourier integrals and makes with them bold transformations, or considers the limit $\nu, \varepsilon \rightarrow 0$ simultaneously with the limit $L \rightarrow \infty$. That is, considers the iterated limit

$$
L \rightarrow \infty, \quad \varepsilon, \nu \rightarrow 0
$$

and treats it in an equally bold way. Concerning this limit the WT makes a number of remarkable predictions, based on tools and ideas, developed in the community, which can be traced back to the work [Pei97]. Relation between the parameters in (0.9) is not quite clear, and it may be better to talk about the WT limits (rather then about a single case).

In order to understand the double limit above, it is natural to study first the limit $\nu \rightarrow 0$ (with $L$ fixed). Its deterministic version recently got attention in physical literature as the "discrete turbulence (DT) limit", see [Kar10] and [Naz11], Section 10. Similar limits were considered by mathematicians, interested in related problems (see [GG12]), and were used by them for intermediate arguments (e.g., see [FGH15]).

Our work is dedicated to rigorous justification of the DT limit for the damped-driven equation $(0.3)_{\nu \sim \varepsilon^{2 q_{*}}}=(0.5)$. Namely, we show that when $\nu \rightarrow 0$, statistical characteristics of actions of solution $u^{\nu}$ for $(0.5)$ have limits of order one, described by actions of solutions for a certain effective equation which is a nonlinear stochastic equation with coefficients of order one and with a hamiltonian nonlinearity, made out of the resonant terms of the nonlinearity $|u|^{2 q_{*}} u$.

The effective equation above is a natural stochastic version of similar equations from the deterministic (physical) DT (see [Kar10, Naz11]). So, in a sense, our results justify the physical DT in the stochastic setting. But in the stochastic case we do more than that since we also treat the stationary regime for eq. (0.5) and show that it converges to that for the effective equation. So solutions of the latter approximate (in distribution) solutions of the former as $t \rightarrow \infty$ and $\nu \rightarrow 0$. Remarkably, in the stationary regime the effective equation approximates not only the actions of solutions with $\nu \ll 1$, but also their angles, see below.

As the title of the paper suggests, our argument is a form of averaging. The latter is a tool which is used by the WT community on a regular basis, either explicitly (e.g. see [Naz11]), or implicitly.

\subsection{Inviscid limits for damped/driven hamiltonian PDE, effective equations and interaction representation}

Equation (0.3) is the linear HPDE (=linear hamiltonian PDE) $(0.1)_{\varepsilon=0}$, driven by the random force, damped by the linear damping $-\nu f(-\Delta u)$ and perturbed by the hamiltonian nonlinearity $-\varepsilon^{2 q_{*}} i \rho|u|^{2 q_{*}} u$. Damped/driven HPDE and the inviscid limits in these equations when the random force and the damping go to zero, are very important for physics. In particular, since the $d$-dimensional Navier-Stokes equation (NSE) with a random force can be regarded as a damped/driven Euler equation (which is an HPDE), and the inviscid limit for the NSE describes the $d$-dimensional turbulence. The NSE with a random force, especially when $d=2$, was intensively studied last years, but the corresponding inviscid limit turned out to be very complicated even for $d=2$, see [KS12]. The problem of this limit becomes feasible when the underlying HPDE is integrable or linear. The most famous integrable PDE is the KdV equation. Its damped/driven perturbations and the corresponding inviscid limits were studied 
in [KP08, Kuk10]. In [Kuk13] the method of those works was applied to the situation when the unperturbed HPDE is the Schrödinger equation

$$
u_{t}+i(-\Delta u+V(x) u)=0, \quad x \in \mathbb{T}_{L}^{d},
$$

where the potential $V(x)$ is in general position. Crucial for the just mentioned works is that there the unperturbed equation is free from strong resonances. For [KP08, Kuk10] it means that all solutions of $\mathrm{KdV}$ are almost-periodic functions of time, such that for a typical solution the corresponding frequency vector is free from resonances; while for [Kuk13] it means that for the typical potentials $V(x)$, considered in [Kuk13], the spectrum of the linear operator in $(0.10)$ is non-resonant.

In contrast, now the linear operator in the unperturbed equation $(0.1)_{\varepsilon=0}$ has the eigenvalues $\lambda_{\mathbf{k}} \in \mathbf{k}^{-2} \mathbb{Z}, \mathbf{k} \in \mathbb{Z}_{L}^{d}$ (see (0.4)), which are highly resonant (accordingly, all solutions for eq. $(0.1)_{\varepsilon=0}$ are periodic with the same period $\left.2 \pi L^{-2}\right)$. This gives rise to an additional difficulty. To explain it, we rewrite equation $(0.5)=(0.6)$ as a fast-slow system, denoting $I_{\mathbf{k}}=\frac{1}{2}\left|v_{\mathbf{k}}\right|^{2}, \quad \varphi_{\mathbf{k}}=$ $\operatorname{Arg} v_{\mathbf{k}}$ (these are the action-angles for the linear hamiltonian system $\left.(0.1)_{\varepsilon=0}\right)$. In the new variables eq. (0.5) reads

$$
\begin{gathered}
\dot{I}_{\mathbf{k}}(\tau)=v_{\mathbf{k}} \cdot P_{\mathbf{k}}(v)+b_{\mathbf{k}}^{2}+b_{\mathbf{k}}\left(v_{\mathbf{k}} \cdot \dot{\boldsymbol{\beta}}^{\mathbf{k}}\right), \\
\dot{\varphi}_{\mathbf{k}}(\tau)=-\nu^{-1} \lambda_{\mathbf{k}}+I_{\mathbf{k}}^{-1} \ldots,
\end{gathered}
$$

where $\mathbf{k} \in \mathbb{Z}_{L}^{d}$, the dot $\cdot$ indicates the real scalar product in $\mathbb{C} \simeq \mathbb{R}^{2}, P(v)$ is the vector field in the r.h.s. of the $v$-equation (0.6) and ... abbreviates a factor of order one (as $\nu \rightarrow 0)$. If the frequencies $\left\{\lambda_{\mathbf{k}}\right\}$ are resonant, then equations for some linear combinations of the phases $\varphi_{\mathbf{k}}$ are slow, which make it more difficult to analyze the system. The method of resonant averaging treats this problem in finite dimension, see [AKN06] and Section 1.2 below. In the situation at hand, we have additional problem: the $\varphi$-equations (0.12) have singularities at the locus

$$
\partial=\left\{I: I_{\mathbf{k}}=0 \text { for some } \mathbf{k}\right\}
$$

which is dense in the space of sequences $\left(I_{\mathbf{k}}, \mathbf{k} \in \mathbb{Z}_{L}^{d}\right)$, and the averaged $I$-equations

$$
\dot{I}_{\mathbf{k}}(\tau)=\left\langle v_{\mathbf{k}} \cdot P_{\mathbf{k}}\right\rangle(I)+b_{\mathbf{k}}^{2}+b_{\mathbf{k}} \sqrt{2 I_{\mathbf{k}}} \dot{\beta}^{\mathbf{k}}(\tau), \quad \mathbf{k} \in \mathbb{Z}_{L}^{d},
$$

where $\langle\cdot\rangle$ signifies the average in $\varphi \in \mathbb{T}^{\infty}$, have there weak singularities. A way to overcome these difficulties is to find for $(0.11),(0.12)$ an effective equation, which is a system of regular equations

$$
\dot{v}_{\mathbf{k}}=R_{\mathbf{k}}(v)+b_{\mathbf{k}} \dot{\boldsymbol{\beta}}^{\mathbf{k}}(\tau), \quad \mathbf{k} \in \mathbb{Z}_{L}^{d},
$$

such that under the natural projection $v_{\mathbf{k}} \mapsto I_{\mathbf{k}}=\frac{1}{2}\left|v_{\mathbf{k}}\right|^{2}, \mathbf{k} \in \mathbb{Z}_{L}^{d}$, solutions of (0.15) transform to solutions of (0.14). In [Kuk10] this approach was used to study the perturbed KdV equation, written as a fast-slow system, similar to (0.11), (0.12). That system has strongly non-linear behaviour, and in [Kuk10] the effective equation was constructed as a kind of averaging of the corresponding $I$-equations. In [Kuk13] an effective equation for the damped/driven nonresonant equation (0.10) was derived in a similar way. If the introduced damping is linear and the nonlinearity is hamiltonian, like in eq. (0.3), then the effective equation in [Kuk13] is linear. 
When the unperturbed hamiltonian system is linear, an alternative way to find an effective equation is to use the interaction representation. I.e., to pass from the complex variables $v_{\mathbf{k}}(\tau)$ (which diagonalise the linear system) to the fast rotating variables

$$
a_{\mathbf{k}}(\tau)=e^{i \nu^{-1} \lambda_{\mathbf{k}} \tau} v_{\mathbf{k}}(\tau), \quad \mathbf{k} \in \mathbb{Z}_{L}^{d} .
$$

Since $\left|a_{\mathbf{k}}\right|=\left|v_{\mathbf{k}}\right|$, then the limiting dynamics of the $a$-variables controls the limiting behaviour of the actions $I_{\mathbf{k}}$. So a regular system of equations, describing the limiting $a$-dynamics, is the effective equation. N. N. Bogolyubov used this approach for the finite-dimensional deterministic averaging, calling it averaging in the quasilinear systems (see in [AKN06]). The interaction representation is systematically used in the WT.

Now consider the fast-slow equations $(0.11),(0.12)$ which come from eq. (0.6), where the fast motion (0.12) is highly resonant. Repeating the construction of the effective equation from [Kuk13], but replacing there the usual averaging by the resonant averaging, we find an effective equation, corresponding to (0.6). It turned out to be another damped/driven hamiltonian system with a Hamiltonian $\mathcal{H}^{\text {res }}$, obtained by the resonant averaging of $\mathcal{H}(v)$, see Section 2.2. As we said above, an alternative way to derive the effective equation is through the interaction representation, i.e., by transition from the $v$-variables to the $a$-variables (0.16). In view of (0.6), the $a$-variables satisfy the system of equations

$$
\begin{aligned}
\dot{a}_{\mathbf{k}}= & -\gamma_{\mathbf{k}} a_{\mathbf{k}}+e^{i \nu^{-1} \lambda_{\mathbf{k}} \tau} b_{\mathbf{k}} \dot{\beta}^{\mathbf{k}}(\tau) \\
& -\rho i \sum_{\mathbf{k}_{1}, \ldots \mathbf{k}_{2 q_{*}+1} \in \mathbb{Z}_{L}^{d}} a_{\mathbf{k}_{1}} \ldots a_{\mathbf{k}_{q_{*}+1}} \bar{a}_{\mathbf{k}_{q_{*}+2}} \ldots \bar{a}_{\mathbf{k}_{2 q_{*}+1}} \delta_{q_{*}+2 \ldots 2 q_{*}+1 \mathbf{k}}^{1 \ldots q_{*}+1} \\
& \times \exp \left(-i \nu^{-1} \tau\left(\lambda_{\mathbf{k}_{1}}+\cdots+\lambda_{\mathbf{k}_{q_{*}+1}}-\lambda_{\mathbf{k}_{q_{*}+2}}-\cdots-\lambda_{\mathbf{k}_{2 q_{*}+1}}-\lambda_{\mathbf{k}}\right)\right), \quad \mathbf{k} \in \mathbb{Z}_{L}^{d} .
\end{aligned}
$$

The terms, constituting the nonlinearity, oscillate fast as $\nu$ goes to zero, unless the sum of the eigenvalues in the exponent in the third line vanishes. The processes $\left\{e^{i \nu^{-1} \lambda_{\mathbf{k}} \tau} \dot{\beta}^{\mathbf{k}}(\tau), \mathbf{k} \in \mathbb{Z}_{L}^{d}\right\}$, make another set of standard independent complex white noises. This leads to the right guess that only the terms for which this sum equals zero (i.e., the resonant terms), contribute to the limiting dynamics, and that the effective equation is the following damped/driven hamiltonian system

$$
\dot{v}_{\mathbf{k}}=-\gamma_{\mathbf{k}} v_{\mathbf{k}}+2 \rho i \frac{\partial \mathcal{H}^{\mathrm{res}}(v)}{\partial \bar{v}_{\mathbf{k}}}+b_{\mathbf{k}} \dot{\beta}^{\mathbf{k}}(\tau), \quad \mathbf{k} \in \mathbb{Z}_{L}^{d} .
$$

Here the Hamiltonian $\mathcal{H}^{\text {res }}(v)$ is given by the sum

$$
-\frac{1}{2 q_{*}+2} \sum_{\mathbf{k}_{1}, \ldots \mathbf{k}_{2 q_{*}+2} \in \mathbb{Z}_{L}^{d}} v_{\mathbf{k}_{1}} \ldots v_{\mathbf{k}_{q_{*}+1}} \bar{v}_{\mathbf{k}_{q_{*}+2}} \ldots \bar{v}_{\mathbf{k}_{2 q_{*}+2}} \delta_{q_{*}+2 \ldots 2 q_{*}+2}^{1 \ldots q_{*}+1} \delta\left(\lambda_{q_{*}+2 \ldots 2 q_{*}+2}^{1 \ldots q_{*}+1}\right),
$$

so that $2 \rho i \frac{\partial \mathcal{H}^{\text {res }}}{\partial \bar{v}_{\mathbf{k}}}(v)$ is

$$
-\rho i \sum_{\mathbf{k}_{1}, \ldots \mathbf{k}_{2 q_{*}+2} \in \mathbb{Z}_{L}^{d}} v_{\mathbf{k}_{1}} \ldots v_{\mathbf{k}_{q_{*}+1}} \bar{v}_{\mathbf{k}_{q_{*}+2}} \ldots \bar{v}_{\mathbf{k}_{2 q_{*}+1}} \delta_{q_{*}+2 \ldots 2 q_{*}+1}^{1 \ldots q_{*}+1} \delta\left(\lambda_{q_{*}+2 \ldots 2 q_{*}+1 \mathbf{k}}^{1 \ldots q_{*}+1}\right),
$$

where we use another physical abbreviation:

$$
\delta\left(\lambda_{q_{*}+2 \ldots 2 q_{*}+2}^{1 \ldots q_{*}+1}\right)=\left\{\begin{array}{cc}
1 & \text { if } \lambda_{\mathbf{k}_{1}}+\ldots+\lambda_{\mathbf{k}_{q_{*}+1}}-\lambda_{\mathbf{k}_{q_{*}+2}}-\ldots-\lambda_{\mathbf{k}_{2 q_{*}+2}}=0, \\
0 & \text { otherwise. }
\end{array} .\right.
$$


This representation for $\mathcal{H}^{\text {res }}$ is different from that given by the resonant averaging. Its advantage is the natural relation with the $a$-variables, which is convenient to study the limit $\nu \rightarrow 0$. The representation for $\mathcal{H}^{\text {res }}$ by means of the resonant averaging turned out to be more useful to study properties of $\mathcal{H}^{\text {res }}$ and of the corresponding hamiltonian vector field.

We saw that the effective equation can be obtained from the system (0.6) by a simple procedure: drop the fast rotations and replace the Hamiltonian $\mathcal{H}$ by its resonant average $\mathcal{H}^{\text {res }}$. In difference with the non-resonant case, this is a nonlinear system. The corresponding hamiltonian system

$$
\dot{v}_{\mathbf{k}}=2 \rho i \frac{\partial \mathcal{H}^{\mathrm{res}}(v)}{\partial \bar{v}_{\mathbf{k}}}, \quad \mathbf{k} \in \mathbb{Z}_{L}^{d},
$$

has a vector field, locally Lipschitz in sufficiently smooth spaces, so equation (0.18) is well posed locally in time. In fact, it is globally well posed. We get this result in Section 4.1 as a simple consequence of our main theorems.

The Hamiltonian $\mathcal{H}^{\text {res }}$ has two convex quadratic integrals,

$$
H_{0}(v)=\frac{1}{2} \sum\left|v_{\mathbf{k}}\right|^{2}, \quad H_{1}=\frac{1}{2} \sum \lambda_{\mathbf{k}}\left|v_{\mathbf{k}}\right|^{2},
$$

which are similar to the energy and the enstrophy integrals for the $2 \mathrm{~d}$ Euler equation on $\mathbb{T}^{2}$ (see (2.32)), and the vector-integral of moments $M(u)=\frac{1}{2} \sum \mathbf{k}\left|u_{\mathbf{k}}\right|^{2} \in \mathbb{R}^{d}$, which can be compared with the extra integrals of the $2 \mathrm{~d}$ Euler. Besides, the vector-field (0.20) is non-linear homogeneous and hamiltonian, as that of the Euler equation. This makes the effective equation (0.18) similar to the $2 \mathrm{~d}$ Navier-Stokes system on $\mathbb{T}^{2}$. Fortunately the former is significantly simpler then the latter.

\section{$0.4 \quad$ Results}

Main results of our work are stated and proved in Section 4, based on properties of the effective equation, established earlier. They imply that the long-time behaviour of solutions for equations (0.5), when $\nu \rightarrow 0$, is controlled in distribution by solutions for the effective equation. We start with the results on the Cauchy problem. So, let $v^{\nu}(\tau)$ be a solution of $(0.6)$ such that $v^{\nu}(0)=v_{0}$, where $v_{0}=\left(v_{0 \mathbf{k}}, \mathbf{k} \in \mathbb{Z}_{L}^{d}\right)$ corresponds to a sufficiently smooth function $u_{0}(x)$. Let us fix any $T>0$.

Consider the vector of actions $I\left(v^{\nu}(\tau)\right)=\left\{I_{\mathbf{k}}\left(v^{\nu}(\tau)\right), \mathbf{k} \in \mathbb{Z}_{L}^{d}\right\}$.

Theorem 1. When $\nu \rightarrow 0$, we have the weak convergence of measures

$$
\mathcal{D}\left(I\left(v^{\nu}(\tau)\right)\right) \rightarrow \mathcal{D}\left(I\left(v^{0}(\tau)\right)\right),
$$

where $v^{0}(\tau), 0 \leq \tau \leq T$, is a unique solution of equation (0.18) such that $v^{0}(0)=v_{0}$.

For any $\xi \in \mathbb{Z}_{0}^{\infty}$, where $\mathbb{Z}_{0}^{\infty}$ is the set of integer vectors $\left(\xi_{\mathbf{k}}, \mathbf{k} \in \mathbb{Z}_{L}^{d}\right)$ of finite length, we denote $\Phi^{\xi}\left(v^{\nu}(\tau)\right):=\sum \xi_{\mathbf{k}} \varphi_{\mathbf{k}}\left(v^{\nu}(\tau)\right) \in S^{1}=\mathbb{R} / 2 \pi \mathbb{Z}$. Then, in addition to (0.23), for a resonant vector $\xi \in \mathbb{Z}_{0}^{\infty}{ }^{3}$ the distribution of $\Phi^{\xi}\left(v^{\nu}(\tau)\right)$, mollified in $\tau$, converges as $\nu \rightarrow 0$ to that of $\Phi^{\xi}\left(v^{0}(\tau)\right)$. On the contrary, if $\xi$ is non-resonant, then the measure $\mathcal{D}\left(\Phi^{\xi}\left(v^{\nu}(\tau)\right)\right.$, mollified in $\tau$, converges to the Lebesgue measure on $S^{1}$. All this is proved in Section 4.1, using the interaction representation (0.17) for equation (0.3).

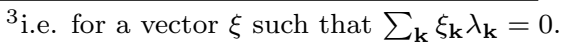


The limiting behaviour of solutions $v^{\nu}(\tau)$ can be described without evoking the effective equation. See Proposition 4.5.

Now consider a stationary measure $\mu^{\nu}$ for equation (0.5) (it always exist). We have Theorem 2. Every sequence $\nu_{j}^{\prime} \rightarrow 0$ has a subsequence $\nu_{j} \rightarrow 0$ such that

$$
I \circ \mu^{\nu_{j}} \rightarrow I \circ m^{0}, \quad \Phi^{(\xi)} \circ \mu^{\nu_{j}} \rightarrow \Phi^{(\xi)} \circ m^{0}
$$

for any resonant vector $\xi \in \mathbb{Z}_{0}^{\infty}$, where $m^{0}$ is a stationary measure for equation (0.18). If a vector $\xi$ is non-resonant, then the measure $\Phi^{\xi} \circ \mu^{\nu}$ converges, as $\nu \rightarrow 0$, to the Lebesgue measure on $S^{1}$.

If the effective equation has a unique stationary measure $m^{0}$, then the limits in Theorem 2 do not depend on the sequence $\nu_{j} \rightarrow 0$, so the convergences hold as $\nu \rightarrow 0$. Remarkably, in this case the measure $m^{0}$ controls not only the slow, but also the fast components of the measures $\mu^{\nu}$ :

Theorem 3. If the effective equation has a unique stationary measure $m^{0}$, then $\mu^{\nu} \rightarrow m^{0}$ as $\nu \rightarrow 0$.

In particular, if the effective equation has a unique stationary measure $m^{0}$ and the equation $(0.3)$ is mixing, ${ }^{4}$ then $m^{0}$ describes asymptotical behaviour of distributions of solutions $u(t)$ for $(0.3)$ as $t \rightarrow \infty$ and $\nu \rightarrow 0$ :

$$
\lim _{\nu \rightarrow 0} \lim _{t \rightarrow \infty} \mathcal{D}(u(t))=m^{0} .
$$

In view of the last theorem, it is important to understand when the effective equation has a unique stationary measure and is mixing. This is discussed in Section 4.3. In particular, the mixing holds if $q_{*}=1, f(t)=t+1$ and $d \leq 3$.

Other equations. Our approach applies to other equations, usually considered in the WT. In particular, in [KM15b] we apply it to the $2 \mathrm{~d}$ quasigeostrophic equation on the $\beta$-plane with random force:

$$
(-\Delta+K) \psi-\rho J(\psi, \Delta \psi)-\beta \psi_{x}=\langle\text { random force }\rangle-\kappa \Delta^{2} \psi+\Delta \psi .
$$

Here $\psi$ is the stream function, $\psi=\psi(t, x, y)$, where $x \in \mathbb{R} / L \mathbb{Z}$ and $y \in \mathbb{R} / \mathbb{Z} ; \Delta \psi$ is the Ekman damping, $-\kappa \Delta^{2} \psi$ is the kinematic viscosity and the random force is similar to that in eq. $(0.3)=(0.5)$. The equation has the same structure as $(0.5)$, and our approach applies to prove that for typical values of the horizontal period $L$ (when the structure of resonances is relatively simple) the limiting, as $\beta \rightarrow \infty$, behaviour or solutions for (0.24) exists, is uniform in $\kappa \in(0,1]$, and is described by an effective equation which is an infinite system of stochastic equations. This system splits to invariant systems of complex dimension $\leq 3$; each of them is an integrable hamiltonian system, coupled with a Langevin thermostat. Under the iterated limits $\lim _{L=\rho \rightarrow \infty} \lim _{\beta \rightarrow \infty}$ and $\lim _{\kappa \rightarrow 0} \lim _{\beta \rightarrow \infty}$ we get similar systems. In particular, none of the three limiting systems exhibits the energy cascade to high frequencies.

\footnotetext{
${ }^{4}$ both these conditions hold, e.g. if $q_{*}=1$ and $f(\lambda)=c_{1}+\lambda^{c_{d}}$, where $c_{d}$ is sufficiently big in terms of $d$.
} 


\section{$0.5 \quad$ Weak Turbulence}

The most famous prediction of the WT (see Section 0.2) deals with the distribution of the energy of solutions for $(0.1)$ and $(0.3)$ between the frequencies. To describe the corresponding claims, consider the quantity $\mathbf{E}\left|v_{\mathbf{k}}(\tau)\right|^{2}$, average it in time ${ }^{5} \tau$ and in wave-vectors $\mathbf{k} \in \mathbb{Z}_{L}^{d}$ such that $|\mathbf{k}| \approx r>0$; next properly scale this and denote the result $E_{r}$. The function $r \rightarrow E_{r}$ is called the energy spectrum. It is predicted by the WT that, in a certain inertial range $r \in\left[r_{1}, r_{2}\right]$, which is contained in the spectral zone where the random force is negligible (i.e., where $\left.\left|b_{\mathbf{k}}\right| \lll\left(\mathbf{E}\left|v_{\mathbf{k}}\right|^{2}\right)^{1 / 2}\right)$, the energy spectrum has an algebraic behaviour:

$$
E_{r} \sim r^{-\alpha} \text { for } r \in\left[r_{1}, r_{2}\right],
$$

for a suitable $\alpha>0$. If the WT is stated in terms of the iterated limits (0.9), then only the limits which lead to the algebraic energy spectra (0.25) are relevant.

In our sequel work [KM15a] we study the effective equation for (0.5) under the limit $L \rightarrow \infty$, evoking the heuristic tools from WT, as presented in [ZLF92], and mimicking the logic of that book. There we show (heuristically) that a suitable choice of the function $\rho(L)$ leads, in the limit of $L \rightarrow \infty$, to a wave kinetic equation for the averaged actions $n_{\mathbf{k}}(t)=\frac{1}{2} \mathbf{E}\left|v_{\mathbf{k}}(t)\right|^{2}$. That equation is different from the heuristic kinetic equations, obtained by the WT methods (see [CFG08], sec. 1.2, and [ZLF92], sec. 2.2.3), but is closely related to them, so that the Zakharov ansatz applies and allows to obtain stationary solutions of the equation, algebraic in $r=|\mathbf{k}|$ and corresponding to energy spectra of the desired form $(0.25) .{ }^{6}$

The rigorous and heuristic results, obtained in this work and in [KM15a], encourage us to pursue our program to study the WT in the model, given by eq. (0.3), which brings to the WT the advantage of a rigorous foundation, based on the recent results of stochastic calculus. We believe that some predictions of the WT (including the fact that the limiting behaviour of the averaged actions $n_{\mathbf{k}}(t)$ is described by a certain wave kinetic equation which admits stationary solutions of the algebraical form (0.25)), may be obtained if not under the iterated limit "first $\nu \rightarrow 0$, next $L \rightarrow \infty$ ", then under its suitable modification (e.g., " $\nu \rightarrow 0$ and $L \rightarrow \infty$ in such a way that $\nu L \rightarrow 0 ")$.

Notation and Agreement. The stochastic terminology we use agrees with [KS91]. All filtered probability spaces we work with satisfy the usual condition (see [KS91]). ${ }^{7}$ Sometime we forget to mention that a certain relation holds a.s.

Spaces of integer vectors. We denote by $\mathbb{Z}_{0}^{\infty}$ the set of vectors in $\mathbb{Z}^{\infty}$ of finite length, and denote $\mathbb{Z}_{+0}^{\infty}=\left\{s \in \mathbb{Z}_{0}^{\infty}: s_{k} \geq 0 \forall k\right\}$. Also see (1.15) and (2.9).

\footnotetext{
${ }^{5}$ Certainly this is not needed if we consider stationary solutions of the equation.

${ }^{6}$ The work [CFG08] mainly treats the systems, where the sources and sinks of energy are well separated, so that there is a large spectral region where the forcing and the dissipation are practically absent and where the kinetic equation does not depend explicitly on the form of the forcing and dissipation. For this reason, the stationary spectra, found in [CFG08] for (0.3), are equal to those obtained by the WT methods for the HPDE (0.1). This explains the difference between the kinetic equation in [CFG08] and that in [KM15a] (which deals with the case when the dissipation is present on the whole spectral range). Contrary to [CFG08], the method of the work [ZL75] applies, at least formally, to all kinds of forcing and damping, so the kinetic equation of that work may depend on the forcing and dissipation.

${ }^{7}$ I.e., the corresponding filtrations $\left\{\mathcal{F}_{t}\right\}$ are continuous from the right, and each $\mathcal{F}_{t}$ contains all negligible sets.
} 
Infinite vectors. For an infinite vector $\xi=\left(\xi_{1}, \xi_{2}, \ldots\right)$ (integer, real or complex) and $N \in \mathbb{N}$ we denote by $\xi^{N}$ the vector $\left(\xi_{1}, \ldots, \xi_{N}\right)$, or the vector $\left(\xi_{1}, \ldots, \xi_{N}, 0, \ldots\right)$, depending on the context. For a complex vector $\xi$ and $s \in \mathbb{Z}_{+0}^{\infty}$ we denote $\xi^{s}=\prod_{j} \xi_{j}^{s_{j}}$.

Norms. We use $|\cdot|$ to denote the Euclidean norm in $\mathbb{R}^{d}$ and in $\mathbb{C} \simeq \mathbb{R}^{2}$, as well as the $\ell_{1}$-norm in $\mathbb{Z}_{0}^{\infty}$. For the norms $|\cdot|_{h^{m}}$ and $|\cdot|_{h_{I}^{m}}$ see (1.13) and below that.

Scalar products. The notation "." stands for the scalar product in $\mathbb{Z}_{0}^{\infty}$, the paring of $\mathbb{Z}_{0}^{\infty}$ with $\mathbb{Z}^{\infty}$, the Euclidean scalar product in $\mathbb{R}^{d}$ and in $\mathbb{C}$. The latter means that if $u, v \in \mathbb{C}$, then $u \cdot v=\operatorname{Re}(\bar{u} v)$. The $L_{2}$-product is denoted $\langle\cdot, \cdot\rangle$, and we also denote by $\langle f, \mu\rangle=\langle\mu, f\rangle$ the integral of a function $f$ against a measure $\mu$.

Max/Min. We denote $a \vee b=\max (a, b), a \wedge b=\min (a, b)$.

Acknowledgments. We wish to thank for discussion and advice Sergey Nazarenko, Anatoli Neishtadt and Vladimir Zeitlin. This work was supported by l'Agence Nationale de la Recherche through the grant STOSYMAP (ANR 2011BS0101501).

\section{Preliminaries}

Since in this work we are not interested in the dependence of the results on $L$, from now on it will be kept fixed and equal to 1, apart from Section 3. There we make explicit calculations, controlling how their results depend on $L$.

\subsection{Apriori estimates.}

In this section we discuss preliminary properties of solutions for (0.5). We found it convenient to parametrise the vectors from the trigonometric basis $\left\{e^{i \mathbf{k} \cdot x}\right\}$ by natural numbers and to normalise them. That is, to use the basis $\left\{e^{j}(x), j \geq 1\right\}$, where

$$
e^{j}(x)=(2 \pi)^{-d / 2} e^{i \mathbf{k} \cdot x}, \quad \mathbf{k}=\mathbf{k}(j) .
$$

The functions $e^{j}(x)$ are eigen-vectors of the Laplacian, $-\Delta e^{j}=\lambda_{j} e^{j}$, so ordered that $0=\lambda_{1}<$ $\lambda_{2} \leq \ldots$. Accordingly eq. (0.5) reads

$$
\dot{u}+i \nu^{-1}(-\Delta u)=-f(-\Delta) u-i \rho|u|^{2 q_{*}} u+\frac{d}{d \tau} \sum_{j=1}^{\infty} b_{j} \beta^{j}(\tau) e^{j}(x),
$$

$u=u(\tau, x)$, where $f(-\Delta) e^{j}=\gamma_{j} e^{j}$ with $\gamma_{j}=f\left(\lambda_{j}\right)$. The processes $\boldsymbol{\beta}^{j}=\beta^{j}+i \beta^{-j}, j \geq 1$, are standard independent complex Wiener processes. The real numbers $b_{j}$ are such that for a suitable sufficiently large even integer $r$ (defined below in (2.13)) we have

$$
B_{r}:=2 \sum_{j=1}^{\infty} \lambda_{j}^{r} b_{j}^{2}<\infty .
$$

By $\mathcal{H}^{p}, p \in \mathbb{R}$, we denote the Sobolev space $\mathcal{H}^{p}=H^{p}\left(\mathbb{T}^{d}, \mathbb{C}\right)$, regarded as a real Hilbert space, and denote by $\langle\cdot, \cdot\rangle$ the real $L^{2}$-scalar product on $\mathbb{T}^{d}$. We provide $\mathcal{H}^{p}$ with the norm $\|\cdot\|_{p}$

$$
\|u\|_{p}^{2}=\sum_{j=1}^{\infty}\left|u_{j}\right|^{2}\left(\lambda_{j} \vee 1\right)^{p} \quad \text { for } u(x)=\sum_{j=1}^{\infty} u_{j} e^{j}(x) .
$$


Let $u(t, x)$ be a solution of $(1.2)$ such that $u(0, x)=u_{0}$. It satisfies standard a-priori estimates which we now discuss, following [Kuk13]. Firstly, for a suitable $\varepsilon_{0}>0$, uniformly in $\nu>0$ one has

$$
\mathbf{E} e^{\varepsilon_{0}\|u(\tau)\|_{0}^{2}} \leq C\left(B_{0},\left\|u_{0}\right\|_{0}\right) \quad \forall \tau \geq 0 .
$$

Assume that

$$
q_{*}<\infty \quad \text { if } d=1,2, \quad q_{*}<\frac{2}{d-2} \quad \text { if } d \geq 3 .
$$

Then, the following bounds on the Sobolev norms of the solution hold for each $2 m \leq r$ and every $n$ :

$$
\begin{gathered}
\mathbf{E}\left(\sup _{0 \leq \tau \leq T}\|u(\tau)\|_{2 m}^{2 n}+\int_{0}^{T}\|u(s)\|_{2 m+1}^{2}\|u(s)\|_{2 m}^{2 n-2} d s\right) \\
\leq\left\|u_{0}\right\|_{2 m}^{2 n}+C(m, n, T)\left(1+\left\|u_{0}\right\|_{0}^{c_{m}, n}\right), \\
\mathbf{E}\|u(\tau)\|_{2 m}^{2 n} \leq C(m, n) \quad \forall \tau \geq 0,
\end{gathered}
$$

where $C(m, n, T)$ and $C(m, n)$ also depend on $B_{2 m}$.

Estimates (1.5), (1.6) are assumed everywhere in our work. As we have explained, they are fulfilled under the assumption (1.4), but if the function $f(t)$ grows super-linearly, then the restriction (1.4) may be weakened.

Relations (1.5) in the usual way (cf. [Hai02, KS04, Oda06, Shi06]) imply that eq. (1.2) is regular in the space $\mathcal{H}^{r}$ in the sense that for any $u_{0} \in \mathcal{H}^{r}$ it has a unique strong solution $u(t, x)$, equal to $u_{0}$ at $t=0$, and satisfying estimates (1.3), (1.5) for any $n$. By the Bogolyubov-Krylov argument, applied to a solution of (1.2), starting from the origin at $t=0$, this equation has a stationary measure $\mu^{\nu}$, supported by the space $\mathcal{H}^{r}$, and a corresponding stationary solution $u^{\nu}(\tau), \mathcal{D} u^{\nu}(\tau) \equiv \mu^{\nu}$, also satisfies (1.3) and (1.6).

\subsection{Resonant averaging}

Let $W \in \mathbb{Z}^{n}, n \geq 1$, be a non-zero integer vector such that its components are relatively prime (so if $W=m V$, where $m \in \mathbb{Z}$ and $V \in \mathbb{Z}^{n}$, then $m= \pm 1$ ). We call the set

$$
\mathcal{A}=\mathcal{A}(W):=\left\{s \in \mathbb{Z}^{n}: W \cdot s=0\right\}
$$

the set of resonances for $W$. This is a $\mathbb{Z}$-module. Denote its rank by $r$. Here and everywhere below the finite-dimensional vectors are regarded as column-vectors.

Lemma 1.1. The rank $r$ equals $n-1$. There exists a system $\zeta^{1}, \ldots, \zeta^{n}$ of integer vectors in $\mathbb{Z}^{n}$ such that $\operatorname{span}_{\mathbb{Z}}\left\{\zeta^{1}, \ldots, \zeta^{n-1}\right\}=\mathcal{A}$, and the $n \times n$ matrix $R=\left(\zeta^{1} \zeta^{2} \ldots \zeta^{n}\right)$ is unimodular (i.e., $\operatorname{det} R= \pm 1$ ).

That is, the vectors $\left(\zeta^{1}, \ldots, \zeta^{n-1}\right)$ make an integer basis of the hyperspace $W^{\perp} \subset \mathbb{R}^{n}$.

Proof. We restrict ourselves to the case when some component of the vector $W$ equals one since this is the result we need below. For the general case and for a more general statement see, for example, [Bou71], Section 7. 
Without loss of generality we assume that $W_{n}=1$. Consider the matrix such that its $n$-th column is $W$ and for $j<n$ the $j$-th column is the vector $e^{j}=\left(e_{1}^{j}, \ldots, e_{n}^{j}\right)^{T}$, where $e_{l}^{j}=\delta_{j, l}$. It is unimodular and transforms the basis vector $e^{n}$ to $W$. Its inverse is an unimodular matrix $B$ such that $B W=e^{n}$. Let $s$ be any vector in $\mathcal{A}$. Since

$$
W \cdot s=0 \Leftrightarrow B W \cdot\left(B^{T}\right)^{-1} s=0 \Leftrightarrow e^{n} \cdot\left(B^{T}\right)^{-1} s=0,
$$

then $\left(B^{T}\right)^{-1} s=\sum_{j=1}^{n-1} m_{j} e^{j}$, where $m_{j}$ 's are some integers. This proves the lemma if we choose $\zeta^{j}=B^{T} e^{j}, j=1, \ldots, n$. Note that the matrix $R$ equals $B^{T}$.

Since $R^{T} W=B W=e^{n}$, then the automorphism of the torus $\mathbb{T}^{n} \rightarrow \mathbb{T}^{n}, \varphi \rightarrow y=R^{T} \varphi$, "resolves the resonances" in the differential equation $\dot{\varphi}=W$ in the sense that it transforms it to the equation

$$
\dot{y}=R^{T} W=(0, \ldots, 0,1)^{T} .
$$

Let us consider a mapping $L=L_{\mathcal{A}}: \mathbb{T}^{n} \rightarrow \mathbb{T}^{n-1}$, "dual to the module $\mathcal{A}$ ":

$$
L: \mathbb{T}^{n} \ni \varphi \rightarrow\left(\varphi \cdot R e^{1}, \ldots, \varphi \cdot R e^{n-1}\right)^{T} \in \mathbb{T}^{n-1} .
$$

The basis $\left\{\eta^{j}=\left(R^{T}\right)^{-1} e^{j}, 1 \leq j \leq n\right\}$, is dual to the basis $\left\{\zeta^{j}=R e^{j}, 1 \leq j \leq n\right\}$, since

$$
\eta^{j} \cdot \zeta^{l}=\left(R^{T}\right)^{-1} e^{j} \cdot R e^{l}=\delta_{j, l} .
$$

Therefore if we decompose $\varphi \in \mathbb{T}^{n}$ in the $\eta$-basis, $\varphi=\sum_{k} y_{k} \eta^{k}=\left(R^{T}\right)^{-1} y$, then $L \varphi=$ $\left(y_{1}, \ldots, y_{n-1}\right)^{T}$. That is,

$$
L \circ\left(R^{T}\right)^{-1}\left(y_{1}, \ldots, y_{n}\right)^{T}=\left(y_{1}, \ldots, y_{n-1}\right)^{T} .
$$

In particular, the fibers of the mapping $L$ are the circles $R\left(\{\mathbf{y}\} \times S^{1}\right)$, where $\mathbf{y}=\left(y_{1}, \ldots, y_{n-1}\right)^{T} \in$ $\mathbb{T}^{n-1}$.

For a continuous function $f$ on $\mathbb{T}^{n}$ we define its resonant average with respect to the integer vector $W$ as the function

$$
\langle f\rangle_{W}(\varphi):=\int_{0}^{2 \pi} f(\varphi+t W) d t
$$

where we have set $d t:=\frac{1}{2 \pi} d t$.

Lemma 1.2. Let $f$ be a $C^{\infty}$-function on $\mathbb{T}^{n}, f(\varphi)=\sum f_{s} e^{i s \cdot \varphi}$. Then

$$
\langle f\rangle_{W}(\varphi)=\sum f_{s} \delta_{0, s \cdot W} e^{i s \cdot \varphi}=\sum_{s \in \mathcal{A}(W)} f_{s} e^{i s \cdot \varphi} .
$$

Proof. It is immediate that (1.12) holds for trigonometrical polynomial. Since for $C^{\infty}$-functions the series in (1.12) converges well, then by continuity the result holds for smooth functions $f$. 


\subsection{Resonant averaging in a Hilbert space}

Consider the Fourier transform for complex functions on $\mathbb{T}^{d}$ which we write as the mapping

$$
\mathcal{F}: \mathcal{H} \ni u(x) \mapsto v=\left(v_{1}, v_{2}, \ldots\right) \in \mathbb{C}^{\infty},
$$

defined by the relation $u(x)=\sum v_{k} e^{k}(x)$. In the space of complex sequences we introduce the norms

$$
|v|_{h^{p}}^{2}=\sum_{k \geq 1}\left|v_{k}\right|^{2}\left(\lambda_{k} \vee 1\right)^{p}, \quad p \in \mathbb{R},
$$

and set $h^{p}=\left\{\left.v|| v\right|_{h^{p}}<\infty\right\}$. Then

$$
|\mathcal{F} u|_{h^{p}}=\|u\|_{p} \quad \forall p .
$$

For $k \geq 1$ let us denote $I_{k}=I\left(v_{k}\right)=\frac{1}{2}\left|v_{k}\right|^{2}$ and $\varphi_{k}=\varphi\left(v_{k}\right)$, where for $v \in \mathbb{C} \varphi(v)=$ $\operatorname{Arg} v \in S^{1}$ if $v \neq 0$, and $\varphi(0)=0 \in S^{1}$. For any $r \geq 0$ consider the mappings

$$
\Pi_{I}: h^{r} \ni v \mapsto I=\left(I_{1}, I_{2}, \ldots\right) \in h_{I+}^{r}, \quad \Pi_{\varphi}: h^{r} \ni v \mapsto \varphi=\left(\varphi_{1}, \varphi_{2}, \ldots\right) \in \mathbb{T}^{\infty} .
$$

Here $h_{I+}^{r}$ is the positive octant $\left\{I: I_{k} \geq 0 \forall k\right\}$ in the space $h_{I}^{r}$, where

$$
h_{I}^{r}=\left\{\left.I|| I\right|_{h_{I}^{r}}=2 \sum_{k}\left(\lambda_{k} \vee 1\right)^{r}\left|I_{k}\right|<\infty\right\} .
$$

Abusing a bit notation we will write $\Pi_{I}(\mathcal{F}(u))=I(u), \Pi_{\varphi}(\mathcal{F}(u))=\varphi(u)$. The mapping $I$ : $\mathcal{H}^{r} \rightarrow h_{I}^{r}$ is 2-homogeneous continuous, while the mapping $\varphi: \mathcal{H}^{r} \rightarrow \mathbb{T}^{\infty}$ is Borel-measurable (the torus $\mathbb{T}^{\infty}$ is given the Tikhonov topology and the corresponding Borel sigma-algebra).

For infinite integer vectors $s=\left(s_{1}, s_{2}, \ldots\right)$ (and only for them) we will write the $l_{1}$-norm of $s$ as $|s|$,

$$
|s|=\sum_{j}\left|s_{j}\right| .
$$

We denote $\mathbb{Z}_{0}^{\infty}=\left\{s \in \mathbb{Z}^{\infty}:|s|<\infty\right\}$, and for a vector $s=\left(s_{1}, s_{2}, \ldots\right) \in \mathbb{Z}_{0}^{\infty}$ write

$$
\Lambda \cdot s=\sum_{k} \lambda_{k} s_{k}, \quad \operatorname{supp} s=\left\{k: s_{k} \neq 0\right\}, \quad\lceil s\rceil=\max \left\{k: s_{k} \neq 0\right\} .
$$

Similar for $\varphi \in \mathbb{T}^{\infty}$ and $s \in \mathbb{Z}_{0}^{\infty}$ we write $\varphi \cdot s=s \cdot \varphi=\sum_{k} \varphi_{k} s_{k} \in S^{1}$.

Let us fix some $m \in \mathbb{N} \cup \infty$ and define the set of resonances of order $m$ for the (integer) frequency-vector $\Lambda=\left(\lambda_{1}, \lambda_{2}, \ldots\right)$ as

$$
\mathcal{A}(\Lambda, m)=\left\{s \in \mathbb{Z}_{0}^{\infty}:|s| \leq m, \Lambda \cdot s=0\right\} .
$$

We will abbreviate $\mathcal{A}(\Lambda)=\mathcal{A}(\Lambda, \infty)=\left\{s \in \mathbb{Z}_{0}^{\infty}: \Lambda \cdot s=0\right\}$.

Let us denote $\mathbb{Z}_{+0}^{\infty}=\left\{s \in \mathbb{Z}_{0}^{\infty}: s_{k} \geq 0 \quad \forall k\right\}$, and consider a series on some space $h^{r}, r \geq 0$ :

$$
F(v)=\sum_{p, q, l \in \mathbb{Z}_{+0}^{\infty}} C_{p q l}(2 I)^{p} v^{q} \bar{v}^{l},
$$


where $I=I(v), C_{p q l}=0$ if $\operatorname{supp} q \cap \operatorname{supp} l \neq \emptyset$ and for $v \in h^{r}, q \in \mathbb{Z}_{+0}^{\infty}$ we write $v^{q}=\prod v_{j}^{q_{j}}$. We assume that the series converges normally in $h^{r}$ in the sense that for each $R>0$ we have

$$
\sum_{p, q, l \in \mathbb{Z}_{+0}^{\infty}}\left|C_{p q l}\right| \sup _{|v|_{h^{r}},|w|_{h^{r}} \leq R}\left|v^{p} w^{p} v^{q} w^{l}\right|<\infty .
$$

Clearly $F(v)=\mathbf{F}(v, \bar{v})$, where $\mathbf{F}$ is a (complex) analytic function on $h^{r} \times h^{r}$. Abusing language and following a physical tradition we will say that $F$ is analytic in $v$ and $\bar{v}$. In particular, $F(v)$ is a real-analytic (so continuous) function of $v$, and the series (1.17) converges absolutely.

The resonant averaging of $F$ can be conveniently defined by introducing, for any $\theta \in \mathbb{T}^{\infty}$, the rotation operator $\Psi_{\theta}$, which is a linear operator in $h^{0}$ :

$$
\Psi_{\theta}(v)=v^{\prime}, \quad v_{k}^{\prime}=e^{i \theta_{k}} v_{k} .
$$

Clearly this is an unitary isomorphism of every space $h^{r}$. Note that $(I \times \varphi)\left(\Psi_{\theta} v\right) \equiv(I(v), \varphi(v)+$ $\theta)$. Using that $\Lambda$ is an integer vector and based on definition (1.11), we give the following

Definition. If a function $F \in C\left(h^{r}\right)$ is given by a normally converging series (1.17), then its resonant average with respect to $\Lambda$ is the function

$$
\langle F\rangle_{\Lambda}(v):=\int_{0}^{2 \pi} F\left(\Psi_{t \Lambda}(v)\right) d t, \quad d t=d t / 2 \pi .
$$

Defining a function $\tilde{F}(I, \varphi)$ by the relation $F(v)=\tilde{F}(I(v), \varphi(v))$, we see that $\langle F\rangle_{\Lambda}(v)=$ $\int_{0}^{2 \pi} \tilde{F}(I, \varphi+t \Lambda) d t$. So this definition well agrees with (1.11).

Consider a monomial $F=(2 I)^{p} v^{q} \bar{v}^{l}$. By Lemma 1.2 we have

$$
\left\langle(2 I)^{p} v^{q} \bar{v}^{l}\right\rangle_{\Lambda}=(2 I)^{p} v^{q} \bar{v}^{l} \delta_{0,(q-l) \cdot \Lambda} .
$$

Now assume that $F$ is given by a normally convergent series (1.17) and has degree $\leq m \leq \infty$ in sense that $C_{p q l}=0$ unless $|q|+|l| \leq m$. Then

$$
\langle F\rangle_{\Lambda}(v)=\sum_{q-l \in \mathcal{A}(\Lambda, m)} C_{p q l}(2 I)^{p} v^{q} \bar{v}^{l}=\sum_{(q-l) \cdot \Lambda=0} C_{p q l}(2 I)^{p} v^{q} \bar{v}^{l} .
$$

If the series (1.17) converges normally, then the series in the r.h.s. above also does. It defines an analytic in $(v, \bar{v})$ function. Note that in view of (1.20)

$\langle F\rangle_{\Lambda}$ is a function of $I_{1}, I_{2} \ldots$ and the variables $\{s \cdot \varphi, s \in \mathcal{A}(\Lambda, m)\}$.

\section{Averaging for equation (1.2).}

Everywhere below $T$ is a fixed positive number.

\subsection{Equation (1.2) in $v$-variables, resonant monomials and combina- tions of phases.}

Let us pass in eq. (1.2) with $u \in \mathcal{H}^{r}, r>d / 2$, to the $v$-variables, $v=\mathcal{F}(u) \in h^{r}$ :

$$
d v_{k}+i \nu^{-1} \lambda_{k} v_{k} d \tau=P_{k}(v) d \tau+b_{k} d \boldsymbol{\beta}^{k}(\tau), \quad k \geq 1 ; \quad v(0)=\mathcal{F}\left(u_{0}\right)=: v_{0} .
$$


Here

$$
P_{k}=P_{k}^{1}+P_{k}^{0},
$$

where $P^{1}$ and $P^{0}$ are, correspondingly, the linear and nonlinear hamiltonian parts of the perturbation. So $P_{k}^{1}$ is the Fourier-image of $-f(-\Delta)$, i.e. $P_{k}^{1}=\operatorname{diag}\left\{-\gamma_{k}, k \geq 1\right\}$, while the operator $P^{0}$ is the mapping $u \mapsto-i \rho|u|^{2 q_{*}} u$, written in the $v$-variables. I.e.,

$$
P^{0}(v)=-i \rho \mathcal{F}\left(|u|^{2 q_{*}} u\right), \quad u=\mathcal{F}^{-1}(v) .
$$

Every component $P_{k}^{0}$ of it is a sum of monomials:

$$
P_{k}^{0}(v)=\sum_{p, q, l \in \mathbb{Z}_{+0}^{\infty}} C_{k}^{p q l}(2 I)^{p} v^{q} \bar{v}^{l}=\sum_{p, q, l \in \mathbb{Z}_{+0}^{\infty}} P_{k}^{0 p q l}(v), \quad k \geq 1,
$$

where $C_{k}^{p q l}=0$ unless $2|p|+|q|+|l|=2 q_{*}+1$ and $|q|=|l|+1$. It is straightforward that $P_{k}^{0}(I, \varphi)$ (see $\left.(1.14)\right)$ is a function of $\varphi=\left(\varphi_{j}, j \geq 1\right)$ of order $2 q_{*}+1$, and that the mapping $P^{0}$ is analytic of polynomial growth:

Lemma 2.1. The nonlinearity $P^{0}$ defines a real-analytic transformation of $h^{r}$ if $r>\frac{d}{2}$. The mapping $P^{0}(v)$ and its differential $d P^{0}(v)$ both have polynomial growth in $|v|_{h^{r}}$.

We will refer to equations (2.1) as to the $v$-equations.

For any $s \in \mathbb{Z}_{0}^{\infty}$ consider the linear combination of phases

$$
\Phi^{s}: h^{0} \rightarrow S^{1}, \quad v \mapsto s \cdot \varphi(v) .
$$

We fix

$$
m=2 q_{*}+2,
$$

and find the corresponding set $\mathcal{A}=\mathcal{A}(\Lambda, m)$ of resonances or order $m$ (see (1.16)). We order vectors in the set $\mathcal{A}$, that is write it as $\mathcal{A}=\left\{s^{(1)}, s^{(2)}, \ldots\right\}$, in such a way that $\left\lceil s^{\left(j_{1}\right)}\right\rceil \leq\left\lceil s^{\left(j_{2}\right)}\right\rceil$ if $j_{1} \leq j_{2}$, and for $N \geq 1$ denote

$$
J(N)=\max \left\{j:\left\lceil s^{(j)}\right\rceil \leq N\right\} .
$$

For any $s^{(j)} \in \mathcal{A}$ consider the corresponding resonant combination of phases $\varphi(v), \Phi_{j}(v)=$ $\Phi^{s_{j}}(v)$, and introduce the Borel-measurable mappings

$$
\begin{aligned}
& h^{r} \ni v \mapsto \Phi=\left(\Phi_{1}, \Phi_{2}, \ldots\right) \in S^{1} \times S^{1} \times \cdots=: \mathcal{T}^{\infty}, \\
& h^{r} \ni v \mapsto(I \times \Phi) \in h_{I+}^{r} \times \mathcal{T}^{\infty} .
\end{aligned}
$$

Note that the system $\Phi$ of resonant combinations is highly over-determined: there are many linear relations between its components $\Phi_{j}$.

Let us pass in eq. (2.1) from the complex variables $v_{k}$ to the action-angle variables $I, \varphi$ :

$$
d I_{k}(\tau)=\left(v_{k} \cdot P_{k}\right)(v) d \tau+b_{k}^{2} d \tau+b_{k}\left(v_{k} \cdot d \boldsymbol{\beta}^{k}\right)
$$

(here $\cdot$ indicates the real scalar product in $\mathbb{C} \simeq \mathbb{R}^{2}$ ), and

$$
d \varphi_{k}(\tau)=\left(-\nu^{-1} \lambda_{k}+\left|v_{k}\right|^{-2}\left(i v_{k} \cdot P_{k}(v)\right)\right) d \tau+\left|v_{k}\right|^{-2} b_{k}\left(i v_{k} \cdot d \boldsymbol{\beta}^{k}\right) .
$$


The equations for the actions are slow, while equations for the angles are fast since $d \varphi_{k} \sim \nu^{-1}$. But the resonant combinations $\Phi_{j}$ of angles satisfy slow equations:

$$
d \Phi_{j}(\tau)=\sum_{k \geq 1} s_{k}^{(j)}\left(\left|v_{k}\right|^{-2}\left(i v_{k} \cdot P_{k}\right) d \tau+\left|v_{k}\right|^{-2} b_{k}\left(i v_{k} \cdot d \boldsymbol{\beta}^{k}\right)\right), \quad j \geq 1 .
$$

Repeating for equations (2.1) and (2.5) the argument from Section 7 in [KP08] (also see Section 6.2 in [Kuk10]), we get low bounds for the norms of the components $v_{k}(\tau)$ of $v(\tau)$ :

Lemma 2.2. Let $v^{\nu}(\tau)$ be a solution of $(2.1)$ and $I^{\nu}(\tau)=I\left(v^{\nu}(\tau)\right)$. Then for any $k \geq 1$ the following convergence holds uniformly in $\nu>0$ :

$$
\int_{0}^{T} \mathbf{P}\left\{I_{k}^{\nu}(\tau) \leq \delta\right\} d \tau \rightarrow 0 \quad \text { as } \delta \rightarrow 0
$$

(the rate of the convergence depends on $k$ ).

Now we define and study corresponding resonant monomials of $v$. For any $s \in \mathbb{Z}_{0}^{\infty}$, vectors $s^{+}, s^{-} \in \mathbb{Z}_{+0}^{\infty}$ such that $s=s^{+}-s^{-}$and $\operatorname{supp} s=\operatorname{supp} s^{+} \cup \operatorname{supp} s^{-}, \operatorname{supp} s^{+} \cap \operatorname{supp} s^{-}=\emptyset$ are uniquely defined. Denote by $V^{s}$ the monomial

$$
V^{s}(v)=v^{s^{+}} \bar{v}^{s^{-}}=\prod_{l} v_{l}^{s_{l}^{+}} \prod_{l} \bar{v}_{l}^{s_{l}^{-}} .
$$

This is a real-analytic function on every space $h^{l}$, and $\varphi\left(V^{s}(v)\right)=\Phi^{s}(v)$. Resonant monomials are the functions ${ }^{8}$

$$
V_{j}(v)=V^{s^{(j)}}(v), \quad j=1,2, \ldots
$$

Clearly they satisfy

$$
I\left(V_{j}(v)\right)=(2 I)^{\frac{1}{2}\left|s^{(j)}\right|}:=\prod_{l}\left(2 I_{l}\right)^{\frac{1}{2}\left|s_{l}^{(j)}\right|}, \quad \varphi\left(V_{j}(v)\right)=\Phi_{j}(v) .
$$

Now consider the mapping

$$
V: h^{l} \ni v \mapsto\left(V_{1}, V_{2}, \ldots\right) \in \mathbb{C}^{\infty},
$$

where $\mathbb{C}^{\infty}$ is given the Tikhonov topology. It is continuous for any $l$. For $N \geq 1$ denote

$$
V^{(N)}(v)=\left(V_{1}, \ldots, V_{J}(v)\right) \in \mathbb{C}^{J},
$$

where $J=J(N)$, see $(2.4)$.

For any $s \in \mathbb{Z}_{0}^{\infty}$, applying the Ito formula to the process $V^{s}(v(\tau))$, we get that

$$
\begin{aligned}
d V^{s}=V^{s}\left(-i \nu^{-1}(\Lambda \cdot s) d \tau\right. & +\sum_{j \in \operatorname{supp} s^{+}} s_{j}^{+} v_{j}^{-1}\left(P_{j}(v) d \tau+b_{j} d \boldsymbol{\beta}_{j}\right) \\
& \left.+\sum_{j \in \operatorname{supp} s^{-}} s_{j}^{-} \bar{v}_{j}^{-1}\left(\bar{P}_{j}(v) d \tau+b_{j} d \overline{\boldsymbol{\beta}}_{j}\right)\right) .
\end{aligned}
$$

\footnotetext{
${ }^{8}$ It may be better to call $V_{j}(v)$ a minimal resonant monomial since for any $l \in \mathbb{Z}_{+0}^{\infty}$ the monomial $I^{l} V_{j}(v)$ also is resonant and corresponds to the same resonance.
} 
If $s=\tilde{s} \in \mathbb{Z}_{0}^{\infty}$ is perpendicular to $\Lambda$, then the first term in the r.h.s. vanishes. So $V^{\tilde{s}}(\tau)$ is a slow process, $d V^{\tilde{s}} \sim 1$. In particular, the processes $d V_{j}, j \geq 1$, are slow.

Estimates (1.5) and equation (2.12) readily imply

Lemma 2.3. For any $j \geq 1$ we have $\mathbf{E}\left|V_{j}(v(\cdot))\right|_{C^{1 / 3}[0, T]} \leq C_{j}(T)<\infty$, uniformly in $0<\nu \leq$ 1.

Let us provide the space $C\left([0, T] ; \mathbb{C}^{\infty}\right)$ with the Tikhonov topology, identifying it with the space $C([0, T] ; \mathbb{C})^{\infty}$. This topology is metrisable by the Tikhonov distance. From now on we fix an even integer $r$,

$$
r \geq \frac{d}{2}+1
$$

and abbreviate

$$
h^{r}=h, \quad h_{I}^{r}=h_{I}, \quad C\left([0, T], h_{I+}\right) \times C\left([0, T], \mathbb{C}^{\infty}\right)=: \mathcal{H}_{I, V} .
$$

We provide $\mathcal{H}_{I, V}$ with Tikhonov's distance, the corresponding Borel $\sigma$-algebra and the natural filtration of the sigma-algebras $\left\{\mathcal{F}_{t}, 0 \leq t \leq T\right\}$.

Let us consider a solution $u^{\nu}(\tau)$ of eq. (1.2), satisfying $u(0)=u_{0}$, denote $v^{\nu}(\tau)=\mathcal{F}\left(u^{\nu}(\tau)\right)$ and abbreviate

$$
I\left(v^{\nu}(\tau)\right)=I^{\nu}(\tau), \quad V\left(v^{\nu}(\tau)\right)=V^{\nu}(\tau) \in \mathbb{C}^{\infty} .
$$

Lemma 2.4. 1) Assume that $u_{0} \in \mathcal{H}^{r}$. Then the set of laws $\mathcal{D}\left(I^{\nu}(\cdot), V^{\nu}(\cdot)\right), 0<\nu \leq 1$, is tight in $\mathcal{H}_{I, V}$.

2) Any limiting measure $\mathcal{Q}$ for the set of laws in 1) satisfies

$$
\begin{gathered}
\mathbf{E}^{\mathcal{Q}}|I|_{C\left([0, T], h_{I}^{r}\right)}^{n} \leq C_{n} \quad \forall n \in \mathbb{N}, \quad \mathbf{E}^{\mathcal{Q}} \int_{0}^{T}|I(\tau)|_{h_{I}^{r+1}} d \tau \leq C^{\prime}, \\
\mathbf{E}^{\mathcal{Q}} e^{\varepsilon_{0}|I(\tau)|_{h_{I}^{0}}} \leq C^{\prime \prime} \quad \forall \tau \in[0, T] .
\end{gathered}
$$

Proof. 1) Due to Lemma 2.3 and the Arzelà Theorem, the laws of processes $V_{j}\left(v^{\nu}(\cdot)\right), 0<\nu \leq 1$, are tight in $C([0, T], \mathbb{C})$, for any $j$. Due to estimates $(1.5)$ with $n=1$ and since the actions $I_{k}^{\nu}$ satisfy slow equations (2.5), the laws of processes $I^{\nu}(\tau)$ are tight in $C\left([0, T], h_{I+}\right)$ (e.g. see in [VF88]). Therefore, for every $N$, any sequence $\nu_{\ell} \rightarrow 0$ contains a subsequence such that the laws $\mathcal{D}\left(I^{\nu}(\cdot), V^{(N)}\left(v^{\nu}(\cdot)\right)\right)$ converges along it to a limit. Applying the diagonal process we get another subsequence $\nu_{\ell}^{\prime}$ such that the convergence holds for each $N$. The corresponding limit is a measure $m^{N}$ on the space $C\left([0, T], h_{I+}\right) \times C([0, T], \mathbb{C})^{J(N)}$. Different measures $m^{N}$ agree, so by Kolmogorov's theorem they correspond to some measure $m$ on the sigma-algebra, generated by cylindric subsets of the space $C\left([0, T], h_{I+}\right) \times C([0, T], \mathbb{C})^{\infty}$, which coincides with the Borel sigma-algebra for that space. It is not hard to check that $\mathcal{D}\left(I^{\nu}(\cdot), V^{\nu}(\cdot)\right) \rightarrow m$ as $\nu=\nu_{\ell}^{\prime} \rightarrow 0$. This proves the first assertion.

2) Estimates (2.14) follow from (1.3), (1.5), the weak convergence to $\mathcal{Q}$ and the Fatou lemma; cf. Lemma 1.2.17 in [KS12]. 


\subsection{Averaged equations, effective equation, interaction representation}

Fix $u_{0} \in \mathcal{H}^{r}$ and consider any limiting measure $\mathcal{Q}^{0}$ for the laws

$$
\mathcal{D}\left(I^{\nu_{\ell}}(\cdot), V^{\nu_{\ell}}(\cdot)\right) \rightarrow \mathcal{Q}^{0} \quad \text { as } \quad \nu_{\ell} \rightarrow 0,
$$

existing by Lemma 2.4. Our goal is to show that the limit $\mathcal{Q}^{0}$ does not depend on the sequence $\nu_{\ell} \rightarrow 0$ and develop tools for its study. We begin with writing down averaged equations for the slow components $I$ and $\Phi$ of the process $v(\tau)$, using the rules of the stochastic calculus (see [Kha68, FW03]), and formally replacing there the usual averaging in $\varphi$ by the resonant averaging $\langle\cdot\rangle_{\Lambda}$. Let us first consider the $I$-equations $(2.5)$. The drift in the $k$-th equation is

$$
b_{k}^{2}+v_{k} \cdot P_{k}=b_{k}^{2}+v_{k} \cdot P_{k}^{1}+v_{k} \cdot P_{k}^{0},
$$

where $v_{k} \cdot P_{k}^{1}=-2 \gamma_{k} I_{k}$ and $v_{k} \cdot P_{k}^{0}(v)=\sum_{p, q, l \in \mathbb{Z}_{+0}^{\infty}} v_{k} \cdot P_{k}^{0 p q l}(v)$, see (2.3). By Section 3 the sum converges normally, so the resonant averaging of the drift is well defined. The dispersion matrix for eq. (2.5) with respect to the real Wiener processes $\left(\beta^{1}, \beta^{-1}, \beta^{2}, \ldots\right)$ is $\operatorname{diag}\left\{b_{k}\left(\operatorname{Re} v_{k} \operatorname{Im} v_{k}\right), k \geq 1\right\}$ (it is formed by $1 \times 2$-blocks). The diffusion matrix equals the dispersion matrix times its conjugated and equals $\operatorname{diag}\left\{b_{k}^{2}\left|v_{k}\right|^{2}, k \geq 1\right\}$. It is independent from the angles, so the averaging does not change it. For its square-root we take $\operatorname{diag}\left\{b_{k} \sqrt{2 I_{k}}\right\}$, and accordingly write the $\Lambda$-averaged $I$-equations as

$$
d I_{k}(\tau)=\left\langle v_{k} \cdot P_{k}\right\rangle_{\Lambda}(I, V) d \tau+b_{k}^{2} d \tau+b_{k} \sqrt{2 I_{k}} d \beta^{k}(\tau), \quad k \geq 1
$$

(see $(1.21))$.

Now consider equations (2.7) for resonant combinations $\Phi_{j}$ of the angles. The corresponding dispersion matrix $D=\left(D_{j k}\right)$ is formed by $1 \times 2$-blocks

$$
D_{j k}=-s_{k}^{(j)} b_{k}\left(2 I_{k}\right)^{-1}\left(\operatorname{Im} v_{k}-\operatorname{Re} v_{k}\right) .
$$

Again the diffusion matrix does not depend on the angles and equals $M=\left(M_{j_{1} j_{2}}\right), M_{j_{1} j_{2}}=$ $\sum_{k} s_{k}^{\left(j_{1}\right)} s_{k}^{\left(j_{2}\right)} b_{k}^{2}\left(2 I_{k}\right)^{-1}$. The matrix $D^{\text {new }}$ with the entries $D_{j k}^{n e w}=s_{k}^{(j)} b_{k}\left(2 I_{k}\right)^{-1 / 2}$ satisfies $\left|D^{\text {new }}\right|^{2}=M$, and we write the averaged equations for $\Phi_{j}$ 's as

$$
d \Phi_{j}(\tau)=\sum_{k \geq 1} s_{k}^{(j)}\left(\frac{\left\langle i v_{k} \cdot P_{k}\right\rangle_{\Lambda}(I, V)}{2 I_{k}} d \tau+\frac{b_{k}}{\sqrt{2 I_{k}}} d \beta^{-k}(\tau)\right), \quad j \geq 1
$$

(we use here Wiener processes, independent from those in eq. (2.16) since the differentials $v_{k} \cdot d \boldsymbol{\beta}^{k}$ and $i v_{k} \cdot d \boldsymbol{\beta}^{k}$, corresponding to the noises in equations (2.5) and (2.6), are independent).

Equations $(2.16),(2.17)$ is a system of stochastic differential equations for the process $(I, V)(\tau)$ since each $\Phi_{j}$ is a function of $I$ and $V_{j}$. It is over-determined as there are linear relations between various $\Phi_{j}$ 's. Besides, eq. (2.16) has a weak singularity at the locus $\partial(h)=\cup_{k}\left\{v \in h: v_{k}=0\right\}$, while eq. (2.17) has there a strong singularity.

Consider a component $\left\langle v_{k} \cdot P_{k}^{0}\right\rangle_{\Lambda}(v)$ of the averaged drift in the equation for $I_{k}$. It may be written as

$$
\left\langle v_{k} \cdot P_{k}^{0}\right\rangle_{\Lambda}(v)=\int_{0}^{2 \pi} v_{k} \cdot\left(e^{-i t \lambda_{k}} P_{k}^{0}\left(\Psi_{t \Lambda}(v)\right)\right) d t=v_{k} \cdot R_{k}^{0}(v)
$$


where we set $R_{k}^{0}(v)=\int_{0}^{2 \pi} e^{-i t \lambda_{k}} P_{k}^{0}\left(\Psi_{t \Lambda}(v)\right) d t$. That is,

$$
R^{0}(v)=\int_{0}^{2 \pi} \Psi_{-t \Lambda} P^{0}\left(\Psi_{t \Lambda} v\right) d t
$$

Repeating the derivation of (1.20) and using that $|q|+|l| \leq m-1$, we see that

$$
R_{k}^{0}(v)=\sum_{\substack{p, q, l \in \mathbb{Z}_{+0}^{\infty} \\ q-l \in \mathcal{A}(\Lambda, m)+e^{k} \\|q|+|l|+1 \leq m}} C_{k}^{p q l}(2 I)^{p} v^{q} \bar{v}^{l}
$$

The relation (2.20) interprets $R^{0}(v)$ as a sum of resonant terms of the mapping $P^{0}(v)$, very much in the spirit of the WT, while (2.19) interpret it a result of the resonant averaging of $P^{0}$.

The vector field $R^{0}$ defines locally-Lipschitz operators in the spaces $h^{p}, p>d / 2$ :

$$
\left|R^{0}(v)-R^{0}(w)\right|_{h^{p}} \leq C_{p}\left(|v|_{h^{p}} \vee|w|_{h^{p}}\right)^{2 q_{*}}|v-w|_{h^{p}}
$$

Indeed, in view of (2.19), for any $v, w$ such that $|v|_{h^{p}},|w|_{h^{p}} \leq R$ we have

$$
\left|\left(R^{0}(v)-R^{0}(w)\right)\right|_{h^{p}} \leq \int_{0}^{2 \pi}\left|\Psi_{-t \Lambda}\left(P^{0}\left(\Psi_{t \Lambda} v\right)-P^{0}\left(\Psi_{t \Lambda} w\right)\right)\right|_{h^{p}} d t
$$

Since $P^{0}(v)=-i \rho \mathcal{F}\left(|\hat{v}|^{2 q_{*}} \hat{v}\right)$, where $\hat{v}=\mathcal{F}^{-1} v$, then denoting $\Psi_{t \Lambda} v=v_{t}$, defining $w_{t}$ similarly and using that the operators $\Psi_{\theta}$ define isometries of $h^{p}$, we bound the r.h.s. of (2.22) by

$$
\begin{aligned}
& \int_{0}^{2 \pi} \mid P^{0}\left(v_{t}\right)-\left.P^{0}\left(w_{t}\right)\right|_{h^{p}} d t=\rho \int_{0}^{2 \pi}\left\|\left|\widehat{v}_{t}\right|^{2 q_{*}} \widehat{v}_{t}-\left|\widehat{w}_{t}\right|^{2 q_{*}} \widehat{w_{t}}\right\|_{p} d t \\
& \leq \rho C_{p} R^{2 q_{*}} \int_{0}^{2 \pi}\left\|\widehat{v_{t}}-\widehat{w_{t}}\right\|_{p} d t \leq \rho C_{p} R^{2 q_{*}}|v-w|_{h^{p}} .
\end{aligned}
$$

Finally we set

$$
R=R^{0}+R^{1}, \quad \text { where } \quad R_{k}^{1}(v)=P_{k}^{1}(v)=-\gamma_{k} v_{k} .
$$

Since $\left\langle v_{k} \cdot P_{k}^{1}\right\rangle_{\Lambda}=\left\langle-\sum 2 \gamma_{k} I_{k}\right\rangle_{\Lambda}=v_{k} \cdot P_{k}^{1}=v_{k} \cdot R_{k}^{1}$, then in view of (2.18) we have

$$
\left\langle v_{k} \cdot P_{k}\right\rangle_{\Lambda}(v)=v_{k} \cdot R_{k}(v) .
$$

For further usage we note that by the same argument, $\left\langle i v_{k} \cdot P_{k}^{0}\right\rangle_{\Lambda}=i v_{k} \cdot R_{k}^{0}$ and $\left\langle i v_{k} \cdot P_{k}^{1}\right\rangle_{\Lambda}=$ $0=i v_{k} \cdot R_{k}^{1}$. So also

$$
\left\langle i v_{k} \cdot P_{k}\right\rangle_{\Lambda}(v)=i v_{k} \cdot R_{k}(v) .
$$

Motivated by the averaging theory for equations without resonances in [Kuk10, Kuk13], we now consider the following effective equation for the slow dynamics in eq. (2.5):

$$
d v_{k}=R_{k}(v) d \tau+b_{k} d \boldsymbol{\beta}^{k}, \quad k \geq 1 .
$$

In difference with the averaged equations (2.16) and (2.17), the effective equation is regular, i.e. it does not have singularities at the locus $\partial(h)$. Since $R^{0}: h \rightarrow h$ is locally Lipschitz, then strong solutions for (2.25) exist locally in time and are unique: 
Lemma 2.5. A strong solution of eq. (2.25) with a specified initial data $v(0)=v_{0} \in h$ is unique, a.s.

The relevance of the effective equation for the study of the long-time dynamics in equations $(1.2)=(2.1)$ is clear from the next lemma:

Lemma 2.6. Let a continuous process $v(\tau) \in h$ be a weak solution of (2.25) such that all moments of the random variable $\max _{0 \leq \tau \leq T}|v(\tau)|_{h}$ are finite. Then $I(v(\tau))$ is a weak solution of (2.16). Let stopping times $0 \leq \tau_{1}<\tau_{2} \leq T$ and numbers $\delta_{*}>0, N \in \mathbb{N}$ be such that

$$
I_{k}(v(\tau)) \geq \delta_{*} \quad \text { for } \tau_{1} \leq \tau \leq \tau_{2} \text { and } k \leq N .
$$

Then the process $\left(I(v(\tau)), \Phi_{j}(v(\tau)), j \leq J(N)\right)$ is a weak solution of the system of averaged equations ${ }^{9}$ (2.16), $(2.17)_{j \leq J}$.

Proof. Let $v(\tau)$ satisfies (2.25). Applying Ito's formula to $I_{k}(v(\tau))$ and $\Phi_{j}(v(\tau)), j \leq J$, we get that

$$
d I_{k}=v_{k} \cdot R_{k} d \tau+b_{k}^{2} d \tau+b_{k} v_{k} \cdot d \boldsymbol{\beta}^{k}
$$

and

$$
d \Phi_{j}=\sum_{k \in \operatorname{supp} s^{(j)}} s_{k}^{(j)}\left(\frac{i v_{k} \cdot R_{k}}{\left|v_{k}\right|^{2}} d \tau+\frac{b_{k}}{\left|v_{k}\right|^{2}} i v_{k} \cdot d \boldsymbol{\beta}^{k}\right) .
$$

Using (2.23) and (2.24) we see that (2.27) has the same drift and diffusion as (2.16). So $I(v(\tau))$ is a weak solution of (2.16) (see [Yor74, MR99]). Similar, for $\tau \in\left[\tau_{1}, \tau_{2}\right]$, in view of (2.24), the process $\left(I, \Phi_{j}, j \leq J\right)$, is a weak solution of the system $(2.16),(2.17)_{j \leq J}$.

Now we show that the effective equation describes the limiting (as $\nu \rightarrow 0$ ) dynamics for the equations of motions, written in the $a$-variables of the interaction representation $(0.16)$. Indeed, let $u^{\nu}(\tau)$ be a solution of eq. (1.2), satisfying $u(0)=u_{0}$. Denote $v^{\nu}(\tau)=\mathcal{F}\left(u^{\nu}(\tau)\right)$ and consider the vector of $a$-variables $a^{\nu}(\tau)=\left(a_{k}^{\nu}(\tau)=e^{i \nu^{-1} \lambda_{k} \tau} v_{k}^{\nu}(\tau), k \geq 1\right)$ (cf. (0.16)). Notice that we obviously have

$$
\left|v^{\nu}(\tau)\right|_{h^{m}} \equiv\left|a^{\nu}(\tau)\right|_{h^{m}} \quad \forall m, \quad I\left(v^{\nu}(\tau)\right) \equiv I\left(a^{\nu}(\tau)\right), \quad V\left(v^{\nu}(\tau)\right) \equiv V\left(a^{\nu}(\tau)\right)
$$

(see $(2.11)$ ). From $(2.1)$ we obtain the following system of equations for the vector $a^{\nu}(\tau)$ :

$$
d a_{k}^{\nu}=\left(R_{k}\left(a^{\nu}\right)+\mathcal{R}_{k}\left(a^{\nu}, \nu^{-1} \tau\right)\right) d \tau+b_{k} e^{i \nu^{-1} \lambda_{k} \tau} d \boldsymbol{\beta}^{k}(\tau), \quad k \geq 1,
$$

where we have denoted

$$
\mathcal{R}_{k}\left(a, \nu^{-1} \tau\right)=\sum_{\substack{p, q, l \in \mathbb{Z}_{+0}^{\infty} \\ q-l-e^{k} \notin \mathcal{A}(\Lambda, m) \\|q|+|l|+1 \leq m}} P_{k}^{0 p q l}(a) \exp \left(-i \nu^{-1} \tau\left(\Lambda \cdot\left(q-l-e^{k}\right)\right)\right) .
$$

This is the nonresonant, fast oscillating part of the nonlinearity (because $\left|\Lambda \cdot\left(q-l-e^{k}\right)\right| \geq 1$ ). Since $\left\{\overline{\boldsymbol{\beta}}^{k}(\tau):=\int e^{i \nu^{-1} \lambda_{k} \tau} d \boldsymbol{\beta}^{k}(\tau), k \geq 1\right\}$ is another set of standard independent complex Wiener processes, then the process $a^{\nu}(\tau)$ is a weak solution of the system of equations

$$
d a_{k}^{\nu}=\left(R_{k}\left(a^{\nu}\right)+\mathcal{R}_{k}\left(a^{\nu}, \nu^{-1} \tau\right)\right) d \tau+b_{k} d \boldsymbol{\beta}^{k}(\tau), \quad k \geq 1 .
$$

\footnotetext{
${ }^{9}$ This system is heavily under-determined.
} 
We will refer to equations (2.30) as to the a-equations. It is crucial that they are identical to the effective equation (2.25), apart from terms which oscillate fast as $\nu \rightarrow 0$.

\subsection{Properties of resonant Hamiltonian $\mathcal{H}^{\text {res }}$ and effective equation}

Lemma 2.7. The vector field $R^{0}$ is hamiltonian:

$$
R^{0}=i \rho \nabla \mathcal{H}^{r e s}(v), \quad \forall v \in h^{p}, p>d / 2,
$$

where $\mathcal{H}^{\text {res }}(v)=\langle\mathcal{H}\rangle_{\Lambda}(v)$ and $\mathcal{H}$ is the Hamiltonian (0.2).

Proof. Indeed, since $P^{0}(v)=i \rho \nabla \mathcal{H}(v)$, then

$$
R^{0}(v)=\int_{0}^{2 \pi} \Psi_{-t \Lambda}\left(i \rho \nabla \mathcal{H}\left(\Psi_{t \Lambda}(v)\right)\right) d t=i \rho \nabla_{v} \int_{0}^{2 \pi} \mathcal{H}\left(\Psi_{t \Lambda}(v)\right) d t=i \rho \nabla_{v} \mathcal{H}^{\mathrm{res}}(v),
$$

as $\Psi_{\theta}^{*} \equiv \Psi_{-\theta}$, and where we used (2.19).

Clearly $\mathcal{H}^{\text {res }}(0)=0$. Since $\mathcal{H}(u) \leq-C\|u\|_{0}^{2 q_{*}+2}$ by the Hölder inequality and since the transformations $\Psi_{t \Lambda}$ preserve $\|u\|_{0}$, then

$$
\mathcal{H}^{\mathrm{res}}(u) \leq-C\|u\|_{0}^{2 q_{*}+2} \quad \forall u .
$$

The resonant Hamiltonian $\mathcal{H}^{\text {res }}$ has symmetries, given by some rotations $\Psi_{m}, m \in \mathbb{R}^{\infty}$ :

Lemma 2.8. i) Let $\mathbf{1}=(1,1, \ldots)$. Then $\mathcal{H}^{\text {res }}\left(\Psi_{t \mathbf{1}} v\right)=$ const (i.e., it does not depend on $\left.t\right)$;

ii) Let $\mathcal{M}^{l}$ the $l$-th component of the sequence $(\mathbf{k}(1), \mathbf{k}(2), \ldots), l=1, \ldots, d$ (see (1.1)). Then $\mathcal{H}^{\text {res }}\left(\Psi_{t \mathcal{M}^{l}} v\right)=$ const, for each $l$.

iii) $\mathcal{H}^{\text {res }}\left(\Psi_{t \Lambda} v\right)=$ const.

Proof. i) By (1.19) we have

$$
\mathcal{H}^{\mathrm{res}}\left(\Psi_{t \mathbf{1}} v\right)=\int_{0}^{2 \pi} \mathcal{H}\left(\Psi_{t^{\prime} \Lambda}\left(\Psi_{t \mathbf{1}} v\right)\right) d t^{\prime}=\int_{0}^{2 \pi} \mathcal{H}\left(\Psi_{t \mathbf{1}}\left(\Psi_{t^{\prime} \Lambda} v\right)\right) d t^{\prime}
$$

Let us denote $\Psi_{t \mathbf{1}}\left(\Psi_{t^{\prime} \Lambda} v\right)=v\left(t ; t^{\prime}\right)$. Then $(d / d t) v\left(t ; t^{\prime}\right)=i v$. The flow of this hamiltonian equation commutes with that of the equation with the Hamiltonian $\mathcal{H} .{ }^{10}$ So $\mathcal{H}\left(v\left(t ; t^{\prime}\right)\right)$ is independent from $t$ for each $t^{\prime}$, and i) follows since $\mathcal{H}^{\text {res }}\left(\Psi_{t \mathbf{1}} v\right)=\int \mathcal{H}\left(v\left(t ; t^{\prime}\right)\right) d t^{\prime}$.

ii) Proof is the same since the transformations $\Psi_{t \mathcal{M}^{l}}, t \in \mathbb{R}$, are the flow of the momentum Hamiltonian $M^{l}(u)=\frac{1}{2} \sum_{j=1}^{\infty} \mathbf{k}^{l}(j)\left|u_{j}\right|^{2}$, which commutes with $\mathcal{H}$.

iii) It is a straightforward consequence of the definition (1.19) of the resonant averaging.

Since the transformations $\Psi_{t \mathbf{1}}$ form the flow of the Hamiltonian $H_{0}(v)=\frac{1}{2} \sum\left|v_{j}\right|^{2}=\frac{1}{2}|v|_{h^{0}}^{2}$, the transformations $\Psi_{t \Lambda}$ - the flow of $H_{1}(v)=\frac{1}{2} \sum \lambda_{j}\left|v_{j}\right|^{2}$, and the transformations $\Psi_{t \mathcal{M}^{l}}, t \in \mathbb{R}$ - the flow of the momentum Hamiltonian, we may recast the assertions of the last lemma as follows:

$$
\left\{\mathcal{H}^{\text {res }}, H_{0}\right\}=0, \quad\left\{\mathcal{H}^{\text {res }}, H_{1}\right\}=0, \quad\left\{\mathcal{H}^{\text {res }}, \mathcal{M}^{l}\right\}=0 \quad \forall l .
$$

\footnotetext{
${ }^{10}$ This follows from the fact that the functional $\frac{1}{2}|v|_{h^{0}}^{2}$ is an integral of motion for the Hamiltonian $\mathcal{H}$, which becomes obvious if we note that in the $u$-representation $\mathcal{H}$ has the form $(0.2)$ and $\frac{1}{2}|v|_{h^{0}}^{2}$ is $\frac{1}{2} \int|u|^{2}(x) d x$.
} 
Here $\{\cdot, \cdot\}$ signifies the Poisson bracket. As the transformations $\Psi_{m}, m \in \mathbb{R}^{\infty}$, are symplectic, then the symmetries in the lemma above preserve the hamiltonian vector field $R^{0}$ and commute with it. In particular, since $\Psi_{t \Lambda}=e^{-i t \Delta}$, then the spectral spaces $E_{\lambda}$ of the operator $-\Delta$,

$$
E_{\lambda}=\operatorname{span}\left\{e^{j}: \lambda_{j}=\lambda\right\},
$$

are invariant for the flow-maps of $R^{0}$.

Since the transformations $\Psi_{m}, m \in \mathbb{R}^{\infty}$, obviously preserve the vector field $R^{1}$ as well as the law of the random force in (2.25) (see the proof of the lemma below), then those $\Psi_{m}$ which are symmetries of $R^{0}$ (equivalently, which are symmetries of the Hamiltonian $\mathcal{H}^{\text {res }}$ ), preserve weak solutions of $(2.25)$. So we have:

Lemma 2.9. If $v(\tau)$ is a solution of equation (2.25) and $m \in \mathbb{R}^{\infty}$ be either a vector $m=$ $t \mathbf{1}, t \in \mathbb{R}$, or a vector $m=t \Lambda$, or $m=t \mathcal{M}^{l}, l=1, \ldots, d$, then $\Psi_{m} v(\tau)$ also is a weak solution.

Proof. Denote $\Psi_{m} v(\tau)=v^{\prime}(\tau)$. Applying $\Psi_{m}$ to eq. (2.25), using Lemma 2.8 and exploiting the invariance of the operator $R^{1}$ with respect to $\Psi_{m}$, we get

$$
d v_{k}^{\prime}=\left(\Psi_{m} R(v(\tau))\right)_{k} d \tau+e^{i m_{k}} b_{k} d \boldsymbol{\beta}^{k}=\left(R\left(v^{\prime}(\tau)\right)_{k}+b_{k}\left(e^{i m_{k}} d \boldsymbol{\beta}^{k}\right) .\right.
$$

Since $\left\{e^{i m_{k}} \boldsymbol{\beta}^{k}(\tau), k \geq 1\right\}$ is another set of standard independent Wiener processes, then $v^{\prime}(\tau)$ is a weak solution of $(2.25)$.

Corollary 2.10. If $\mu$ is a stationary measure for equation (2.25) and a vector $m$ is as in Lemma 2.9, then the measure $\Psi_{m} \circ \mu$ also is stationary.

The next lemma characterises the increments of $R^{0}(v)$ in the space $h^{0}$. It will be needed below to study the ergodic properties of the effective equation:

Lemma 2.11. Let $p>d / 2$. Then for any $v, w \in h^{p}$ we have

$$
\left|R^{0}(v)-R^{0}(w)\right|_{h^{0}} \leq C\left(|v|_{h^{p}}+|w|_{h^{p}}\right)^{2 q_{*}}|v-w|_{h^{0}} .
$$

Proof. Repeating the proof of the Lipschitz property of $R^{0}$ in the space $h$ (see (2.21)) and using the notation of that proof, i.e. denoting $\Psi_{t \Lambda} v=v_{t}, \hat{v}=\mathcal{F}^{-1} v$, and similar for the vector $w$, we get that

$$
\begin{aligned}
& \left|R^{0}(v)-R^{0}(w)\right|_{h^{0}} \leq \int_{0}^{2 \pi}\left|\Psi_{-t \Lambda}\left(P^{0}\left(\Psi_{t \Lambda} v\right)-P^{0}\left(\Psi_{t \Lambda} w\right)\right)\right|_{h^{0}} d t \\
& \quad=\int_{0}^{2 \pi}\left|P^{0}\left(v_{t}\right)-P^{0}\left(w_{t}\right)\right|_{h^{0}} d t=\int_{0}^{2 \pi}\left\|\left|\widehat{v}_{t}\right|^{2 q_{*}} \widehat{v_{t}}-\left|\widehat{w}_{t}\right|^{2 q_{*}} \widehat{w_{t}}\right\|_{0} d t \\
& \leq C \int_{0}^{2 \pi}\left(\left|\widehat{v}_{t}\right|_{L^{\infty}}+\left|\widehat{w_{t}}\right|_{L^{\infty}}\right)^{2 q^{*}}\left\|\widehat{v}_{t}-\widehat{w_{t}}\right\|_{0} d t \leq C_{1}\left(|v|_{h^{p}}+|w|_{h^{p}}\right)^{2 q^{*}}|v-w|_{h^{0}} .
\end{aligned}
$$




\section{Explicit calculation}

We intend here to calculate explicitly the effective equation (2.25), keeping track of the dependence on the size $L$ of the torus. To do that, it is convenient to use the natural parametrisation of the exponential basis by vectors $\mathbf{k} \in \mathbb{Z}_{L}^{d}$; that is, decompose functions $u(x)$ to Fourier series, $u(x)=\sum_{\mathbf{k} \in \mathbb{Z}_{L}^{d}} v_{\mathbf{k}} e^{i \mathbf{k} \cdot x}$. We modify the norms $|\cdot|_{h^{p}}$ accordingly :

$$
\|u\|_{p}^{2}=(2 \pi L)^{d} \sum_{\mathbf{k} \in \mathbb{Z}_{L}^{d}}\left(|\mathbf{k}| \vee \frac{1}{L}\right)^{2 p}\left|v_{\mathbf{k}}\right|^{2}=:|v|_{h^{p}}^{2} .
$$

Now, as in the Introduction, the eigenvalues of the minus-Laplacian are $\lambda_{\mathbf{k}}=|\mathbf{k}|^{2}$ and the damping coefficients $\gamma_{\mathbf{k}}=f\left(\lambda_{\mathbf{k}}\right)$.

In the $v$-coordinates the nonlinearity becomes the mapping $v \mapsto P^{0}(v)$, whose $\mathbf{k}$-th component is

$$
P_{\mathbf{k}}^{0}(v)=-i \rho \sum_{\mathbf{k}_{1}, \ldots \mathbf{k}_{2 q_{*}+1} \in \mathbb{Z}_{L}^{d}} v_{\mathbf{k}_{1}} \cdots v_{\mathbf{k}_{q_{*}+1}} \bar{v}_{\mathbf{k}_{q_{*}+2}} \cdots \bar{v}_{\mathbf{k}_{2 q_{*}+1}} \delta_{q_{*}+2 \ldots 2 q_{*}+1 \mathbf{k}}^{1 \ldots q_{*}+1}
$$

(see (0.8)). Accordingly,

$$
v_{\mathbf{k}} \cdot P_{\mathbf{k}}^{0}=\rho \sum_{\mathbf{k}_{1}, \ldots \mathbf{k}_{2 q_{*}+1} \in \mathbb{Z}_{L}^{d}} \operatorname{Im}\left(v_{\mathbf{k}_{1}} \cdots v_{\mathbf{k}_{q_{*}+1}} \bar{v}_{\mathbf{k}_{q_{*}+2}} \cdots \bar{v}_{\mathbf{k}_{2 q_{*}+1}} \bar{v}_{\mathbf{k}}\right) \delta_{q_{*}+2 \ldots 2 q_{*}+1 \mathbf{k}}^{1 \ldots q_{*}+1} .
$$

In order to calculate the resonant average, we first notice that $v_{\mathbf{k}} \cdot P_{\mathbf{k}}^{0}$ can be written as a series (1.17), where $\left|C_{p q l}\right| \leq 1$ and $|q|+|p|+|l|=2 q_{*}+2$. In this case the sum in the l.h.s. of (1.18) is bounded by

$$
C\left(\sum_{\mathbf{k} \in \mathbb{Z}_{L}^{d}}\left|v_{\mathbf{k}}\right|\right)^{2 q_{*}+2} \leq C_{1}(L)|v|_{p}^{q_{*}+1}\left(\sum_{\mathbf{k} \in \mathbb{Z}_{L}^{d}}|\mathbf{k}|^{-2 p}\right)^{q_{*}+1} .
$$

So the condition (1.18) is met if $2 p>d$.

Since the order of the resonance $m=2 q_{*}+2$, then $\left\langle v_{\mathbf{k}} \cdot P_{\mathbf{k}}^{0}\right\rangle_{\Lambda}(v)$ equals

$$
\rho \sum_{\mathbf{k}_{1}, \ldots \mathbf{k}_{2 q_{*}+1} \in \mathbb{Z}_{L}^{d}} \operatorname{Im}\left(v_{\mathbf{k}_{1}} \cdots v_{\mathbf{k}_{q_{*}+1}} \bar{v}_{\mathbf{k}_{q_{*}+2}} \cdots \bar{v}_{\mathbf{k}_{2 q_{*}+1}} \bar{v}_{\mathbf{k}}\right) \delta_{q_{*}+2 \ldots 2 q_{*}+1 \mathbf{k}}^{1 \ldots q_{*}+1} \delta\left(\lambda_{q_{*}+2 \ldots q_{*}+1}^{1}+1,\right.
$$

(see (0.21)). This follows from (3.1) and (1.19) if one notes that appearing there restriction $(q-l) \cdot \Lambda=0$ is now replaced by the factor $\delta\left(\lambda_{q_{*}+2 \ldots 2 q_{*}+1 \mathbf{k}}^{1 \ldots q_{*}+1}\right)$. In a similar way, we see that the quantity $R_{k}^{0}$, entering equation (2.25), takes the form

$$
R_{\mathbf{k}}^{0}(v)=-i \rho \sum_{\mathbf{k}_{1}, \ldots \mathbf{k}_{2 q_{*}+1} \in \mathbb{Z}_{L}^{d}} v_{\mathbf{k}_{1}} \cdots v_{\mathbf{k}_{q_{*}+1}} \bar{v}_{\mathbf{k}_{q_{*}+2}} \cdots \bar{v}_{\mathbf{k}_{2 q_{*}+1}} \delta_{q_{*}+2 \ldots 2 q_{*}+1 \mathbf{k}}^{1 \ldots q_{*}+1} \delta\left(\lambda_{q_{*}+2 \ldots 2 q_{*}+1 \mathbf{k}}^{1 \ldots q_{*}+1}\right) .
$$

Taking into account that $R_{\mathbf{k}}^{1}=-\gamma_{\mathbf{k}} v_{\mathbf{k}}$, we finally arrive at an explicit formula for the effective equation (2.25):

$$
\begin{gathered}
d v_{\mathbf{k}}=\left(-\gamma_{\mathbf{k}} v_{\mathbf{k}}\right. \\
\left.-i \rho \sum_{\mathbf{k}_{1}, \ldots \mathbf{k}_{2 q_{*}+1} \in \mathbb{Z}_{L}^{d}} v_{\mathbf{k}_{1}} \cdots v_{\mathbf{k}_{q_{*}+1}} \bar{v}_{\mathbf{k}_{q_{*}+2}} \cdots \bar{v}_{\mathbf{k}_{2 q_{*}+1}} \delta_{q_{*}+2 \ldots 2 q_{*}+1 \mathbf{k}}^{1 \ldots q_{*}+1} \delta\left(\lambda_{q_{*}+2 \ldots 2 q_{*}+1 \mathbf{k}}^{1 \ldots q_{*}+1}\right)\right) d \tau \\
+b_{\mathbf{k}} d \boldsymbol{\beta}^{\mathbf{k}}, \quad \mathbf{k} \in \mathbb{Z}_{L}^{d} .
\end{gathered}
$$


Due to $(2.31)$,

$$
R_{k}^{0}(v)=i \rho \nabla_{v_{k}} \mathcal{H}^{\mathrm{res}}(v)=2 i \rho \frac{\partial}{\partial \bar{v}_{k}} \mathcal{H}^{\mathrm{res}}(v) .
$$

Therefore eq. (3.2) can be written as the damped-driven hamiltonian system (0.18).

Examples. a) If $q_{*}=1$, then (3.2) reads

$$
d v_{\mathbf{k}}=\left(-\gamma_{\mathbf{k}} v_{\mathbf{k}}-i \rho \sum_{\mathbf{k}, \mathbf{k}^{\prime}, \mathbf{k}^{\prime \prime} \in \mathbb{Z}_{L}^{d}} v_{\mathbf{k}} v_{\mathbf{k}^{\prime}} \bar{v}_{\mathbf{k}^{\prime \prime}} \delta_{\mathbf{k}+\mathbf{k}^{\prime}, \mathbf{k}^{\prime \prime}+r} \delta_{\lambda_{\mathbf{k}}+\lambda_{\mathbf{k}^{\prime}}, \lambda_{\mathbf{k}^{\prime \prime}}+\lambda_{\mathbf{k}}}\right) d \tau+b_{\mathbf{k}} d \boldsymbol{\beta}^{\mathbf{k}},
$$

where $\mathbf{k} \in \mathbb{Z}_{L}^{d}$. If $f(t)=t+1$, then this equation looks similar to the CGL equation

$$
\dot{u}-\Delta u+u=i|u|^{2} u+\frac{d}{d \tau} \sum b_{\mathbf{k}} \boldsymbol{\beta}^{\mathbf{k}}(\tau) e^{i \mathbf{k} \cdot x},
$$

written in the Fourier coefficients. The latter equation possesses nice analytical properties; e.g. its stationary measures is unique for any $d$, see [KN13].

b) Our results remain true if the Hamiltonian $\mathcal{H}$, corresponding to the nonlinearity in (0.5), has variable coefficients. In particular, let $d=1$ and the nonlinearity in $(0.5)$ is replaced by $-i p(x)|u|^{2} u$ with a sufficiently smooth function $p(x)$. Then the effective equation is

$$
d v_{k}=\left(-\gamma_{k} v_{k}-i \sum_{k_{1}, k_{2}, k_{3}, k_{4} \in \mathbb{Z}_{L}} v_{k_{1}} v_{k_{2}} \bar{v}_{k_{3}} p_{k_{4}} \delta_{k_{1}+k_{2}+k_{4}, k_{3}+k} \delta_{k_{1}^{2}+k_{2}^{2}, k_{3}^{2}+k^{2}}\right) d \tau+b_{k} d \boldsymbol{\beta}^{k},
$$

where $k_{L} \in \mathbb{Z}_{d}$ and $p_{k}$ 's are the Fourier coefficients of $p(x)$.

\section{Main results}

\subsection{Averaging theorem for the initial-value problem.}

We recall that $r$ is a fixed even integer such that $r \geq \frac{d}{2}+1$, and abbreviate

$$
h^{r}=h, \quad C([0, T], h)=\mathcal{H}_{a} .
$$

We provide $\mathcal{H}_{a}$ with the Borel $\sigma$-algebra and the natural filtration of the sigma-algebras $\left\{\mathcal{F}_{t}, 0 \leq\right.$ $t \leq T\}$.

Let $v^{\nu}(\tau)$ be a solution of (2.1) such that $v^{\nu}(0)=v_{0}=\mathcal{F}\left(u_{0}\right) \in h^{r}$, consider the corresponding process $a^{\nu}(\tau)$. Due to $(2.28)$, the process $a^{\nu}$ satisfies obvious analogies of the estimates $(1.3),(1.5)$ and $(1.6)$. Since $(R+\mathcal{R})(a)$ is the nonlinearity $P(v)$, written in the $a$-variables, then

$$
|(R+\mathcal{R})(a)(\tau)|_{h}=|P(v)(\tau)|_{h} \leq C|v(\tau)|_{h}^{q_{*}+1}=C|a(\tau)|_{h}^{q_{*}+1} .
$$

Therefore all moments of $|(R+\mathcal{R})(a)|_{\mathcal{H}_{a}}$ are finite, and we get from eq. (2.30) that $\mathbf{E}\left|a^{\nu}\right|_{C^{1 / 3}([0, T], h)} \leq$ $\bar{C}$, uniformly in $\nu$. Now arguing as when proving Lemma 2.4 we get that the set of laws $\mathcal{D}\left(a^{\nu}(\cdot)\right), 0<\nu \leq 1$, is tight in $\mathcal{H}_{a}$. Consider any limiting measure, corresponding to the laws $\mathcal{D}\left(a^{\nu}(\cdot)\right)$ :

$$
\mathcal{D}\left(a^{\nu_{\ell}}(\cdot)\right) \rightarrow \mathcal{Q}_{a}^{0} \quad \text { as } \quad \nu_{\ell} \rightarrow 0 .
$$


Theorem 4.1. There exists a unique weak solution a $(\tau)$ of effective equation (2.25), satisfying $a(0)=v_{0}$ a.s. The law of $a(\cdot)$ in the space $\mathcal{H}_{a}$ coincides with $\mathcal{Q}_{a}^{0}$. The convergence (4.1) holds as $\nu \rightarrow 0$.

The proof of the theorem is presented at the end of this section.

Let $\mathcal{Q}^{0}$ be a measure in $\mathcal{H}_{I, V}$ as in $(2.15)$. Since $(I, V)\left(v^{\nu}(\cdot)\right)=(I, V)\left(a^{\nu}(\cdot)\right)$ for any $\nu>0$ then re-denoting $a(\tau)$ by $v(\tau)$ we derive a corollary from the previous theorem:

Theorem 4.2. There exists a unique weak solution $v(\tau)$ of effective equation (2.25), satisfying $v(0)=v_{0}$ a.s. The law of $(I, V)(v(\cdot))$ in the space $\mathcal{H}_{I, V}$ coincides with $\mathcal{Q}^{0}$ and the convergence (2.15) holds as $\nu \rightarrow 0$. Moreover, for any vectors $\tilde{s}_{1}, \ldots, \tilde{s}_{m} \in \mathbb{Z}_{0}^{\infty}$, perpendicular to $\Lambda$, we have the convergence

$$
\mathcal{D}\left(I, V^{\tilde{s}_{1}}, \ldots, V^{\tilde{s}_{m}}\right)\left(v^{\nu}(\cdot)\right) \rightarrow \mathcal{D}\left(I, V^{\tilde{s}_{1}}, \ldots, V^{\tilde{s}_{m}}\right)(v(\cdot)) .
$$

By this result the Cauchy problem for the effective equation has a weak solution. Using Lemma 2.5 and the Yamada-Watanabe argument (see [KS91, Yor74, MR99]) we get that the equation is well posed:

Corollary 4.3. For any $v_{0} \in h^{r}$, eq. (2.25) has a unique strong and a unique weak solution $v(\tau)$ such that $v(0)=v_{0}$. Its law satisfies (2.14).

Now, let $\tilde{s} \in \mathbb{Z}_{0}^{\infty}$ be any non-zero vector, orthogonal to $\Lambda$, and consider $\varphi\left(v^{\nu}(\tau)\right) \cdot \tilde{s}=$ $\varphi\left(V^{\tilde{s}}\left(v^{\nu}(\tau)\right) \in S^{1}\right.$. Since $\varphi\left(V^{\tilde{s}}\right)$ is a discontinuous function of $V^{\tilde{s}} \in \mathbb{C}$, then to pass to a limit as $\nu \rightarrow 0$ we do the following. We identify $S^{1}$ with $\left\{v \in \mathbb{R}^{2}:|v|=1\right\}$, denote $\lceil\tilde{s}\rceil=N$, and approximate the discontinuous function $V^{N}=\left(V_{1}, \ldots, V_{N}\right) \mapsto \varphi\left(V^{\tilde{s}}\right)$ by continuous functions

$$
V^{N} \mapsto f_{\delta}\left(\left[I\left(V^{N}\right)\right]\right) \varphi\left(V^{\tilde{s}}\right) \in \mathbb{R}^{2}, \quad[I]=\min _{1 \leq k \leq N} I_{k}, \quad 0<\delta \ll 1 .
$$

where $f_{\delta}$ is continuous, $0 \leq f_{\delta} \leq 1, f_{\delta}(t)=0$ for $t \leq \delta / 2$ and $f_{\delta}=1$ for $t \geq \delta$.

For any measure $\mu_{\tau}$ in a complete metric space, which weakly continuously depends on $\tau$, and any $\tau_{1}<\tau_{2}$ we will denote

$$
\left\langle\mu_{\tau}\right\rangle_{\tau_{1}}^{\tau_{2}}=\frac{1}{\tau_{2}-\tau_{1}} \int_{\tau_{1}}^{\tau_{2}} \mu_{\tau} d \tau
$$

Then the argument above jointly with Lemma 2.2 imply:

Corollary 4.4. Let $\tilde{s} \in \mathbb{Z}_{0}^{\infty}$ be any non-zero vector, orthogonal to $\Lambda$, and let $0 \leq \tau_{1}<\tau_{2} \leq T$. Then

$$
\left\langle\mathcal{D}\left(\varphi\left(v^{\nu}(\tau)\right) \cdot \tilde{s}\right)\right\rangle_{\tau_{1}}^{\tau_{2}} \rightarrow\langle\mathcal{D}(\varphi(v(\tau)) \cdot \tilde{s})\rangle_{\tau_{1}}^{\tau_{2}} \quad \text { as } \quad \nu \rightarrow 0
$$

On the contrary, if $s \cdot \Lambda \neq 0$, then by Proposition 4.10 we get that

$$
\left\langle\mathcal{D}\left(\varphi\left(v^{\nu}(\tau)\right) \cdot s\right)\right\rangle_{\tau_{1}}^{\tau_{2}} \rightarrow \Varangle \varphi
$$

More generally, if vectors $\tilde{s}_{1}, \ldots, \tilde{s}_{M}$ from $\mathbb{Z}_{0}^{\infty}$ are perpendicular to $\Lambda$ and a vector $s$ is not, then

$$
\left\langle\mathcal{D}\left(I, \varphi \cdot \tilde{s}_{1}, \ldots, \varphi \cdot \tilde{s}_{M}, \varphi \cdot s\right)\left(v^{\nu}(\tau)\right)\right\rangle_{\tau_{1}}^{\tau_{2}} \rightarrow\left\langle\mathcal{D}\left(I, \varphi \cdot \tilde{s}_{1}, \ldots, \varphi \cdot \tilde{s}_{M}\right)(v(\tau))\right\rangle_{\tau_{1}}^{\tau_{2}} \times đ \varphi
$$

We do not know an equivalent description of the measure $\mathcal{Q}^{0}$ only in terms of the slow variables $(I, V)$ of equation (2.1). But the following result holds true: 
Proposition 4.5. Consider the natural process on the space $\mathcal{H}_{I, V}$ with the measure $\mathcal{Q}^{0}$. If for some $N \in \mathbb{N}$ and $\delta_{*}>0$, stopping times $0 \leq \tau_{1}<\tau_{2} \leq T$ satisfy (2.26), then for $\tau \in\left[\tau_{1}, \tau_{2}\right]$ the process $\left(I, \Phi^{(N)}\right)((I, V)(\tau))$ is a weak solution of the averaged equations (2.16) and (2.17) $\left.\right|_{j \leq J}$. Here $\Phi^{(N)}=\left(\Phi_{1}, \ldots, \Phi_{J(N)}\right)$.

Since the averaged quantities $\left\langle v_{k} \cdot P_{k}\right\rangle_{\Lambda}$ and $\left\langle i v_{k} \cdot P_{k}\right\rangle_{\Lambda}$ are functions of $I$ and $\Phi$ (see (1.21)), then equations (2.16) and (2.17) $\left.\right|_{j \leq J}$ form an under-determined system of equations for the variables $(I, \Phi)$.

Proof of Theorem 4.1 The proof follows the Khasminski scheme (see [Kha68, FW03, KP08]). Its crucial step is given by the following lemma:

Lemma 4.6. For any $k \geq 1$ one has

$$
\mathfrak{A}_{k}^{\nu}:=\mathbf{E} \max _{0 \leq \tau \leq T}\left|\int_{0}^{\tau} \mathcal{R}_{k}\left(a^{\nu}(s), \nu^{-1} s\right) d s\right| \rightarrow 0 \quad \text { as } \nu \rightarrow 0 .
$$

The lemma is proved below in Section 4.4, following the arguments in [KP08, Kuk13]. Now we derive from it the theorem.

For $\tau \in[0, T]$ consider the processes

$$
N_{k}^{\nu_{l}}=a_{k}^{\nu_{l}}(\tau)-\int_{0}^{\tau} R_{k}\left(a^{\nu_{l}}(s)\right) d s, \quad k \geq 1 .
$$

Due to $(2.30)$ we can write $N_{k}^{\nu_{l}}$ as

$$
N_{k}^{\nu_{l}}(\tau)=\widetilde{N}_{k}^{\nu_{l}}(\tau)+\bar{N}_{k}^{\nu_{l}}(\tau)
$$

where $\widetilde{N}_{k}^{\nu_{l}}(\tau)=a^{\nu_{l}}(\tau)-\int_{0}^{\tau}\left(R_{k}\left(a^{\nu_{l}}(s)\right)+\mathcal{R}_{k}\left(a^{\nu_{l}}(s), \nu_{l}^{-1} s\right)\right) d s$ is a $\mathcal{Q}_{a}^{0}$ martingale and the disparity $\bar{N}_{k}^{\nu_{l}}$ is

$$
\bar{N}_{k}^{\nu_{l}}(\tau)=\int_{0}^{\tau} \mathcal{R}_{k}\left(a^{\nu_{l}}(s), \nu_{l}^{-1} s\right) d s .
$$

The convergence $\mathcal{D}\left(a^{\nu_{l}}\right) \rightarrow \mathcal{Q}_{a}^{0}$ and Lemma 4.6 imply that the processes

$$
N_{k}(\tau)=a_{k}(\tau)-\int_{0}^{\tau} R_{k}(a) d s, \quad k \geq 1,
$$

are $\mathcal{Q}_{a}^{0}$ martingales (see for details [KP08], Proposition 6.3).

Similar to (4.2), we find that

$$
\mathbf{E} \max _{0 \leq \tau \leq T}\left|\int_{0}^{\tau} \mathcal{R}_{k}\left(a^{\nu}(s), \nu^{-1} s\right) d s\right|^{2} \rightarrow 0 \quad \text { as } \nu \rightarrow 0 .
$$

Then, using the same arguments as before, we see that the processes $N_{k_{1}}(\tau) N_{k_{2}}(\tau)-\int_{0}^{\tau} A_{k_{1} k_{2}} d s$ are $\mathcal{Q}_{a}^{0}$ martingales, where $A_{k_{1} k_{2}}$ denotes the diffusion matrix for the system (2.25). That is, $\mathcal{Q}_{a}^{0}$ is a solution of the martingale problem with drift $R_{k}$ and the diffusion $A$. Hence, $\mathcal{Q}_{a}^{0}$ is a law of a weak solution of eq. (2.25). Such a solution exists for any $v_{0} \in h$. So by Lemma 2.5 and the Yamada-Watanabe argument (see [KS91, Yor74, MR99]), weak and strong solutions for (2.25) both exist and are unique. Hence, the limit in (2.15) does not depend on the sequence $\nu_{l} \rightarrow 0$, the convergence holds as $\nu \rightarrow 0$, and the theorem is proved. 


\subsection{Averaging theorem for stationary solutions.}

Let $v^{\nu}(\tau)$ be a stationary solution of eq. (2.1) as at the end of Section 1.1. ${ }^{11}$ Solutions $v^{\nu}$ inherit the a-priori estimates (1.3), (1.5), (1.6), so still the set of laws $\mathcal{D}\left(I\left(v^{\nu}(\cdot)\right), V\left(v^{\nu}(\cdot)\right)\right)$, $0<\nu \leq 1$, is tight in $\mathcal{H}_{I, V}$ (cf. Lemma 2.4). Consider any limit

$$
\mathcal{D}\left(I\left(v^{\nu_{\ell}}(\cdot)\right), V\left(v^{\nu_{\ell}}(\cdot)\right)\right) \rightarrow \mathcal{Q} \quad \text { as } \nu_{\ell} \rightarrow 0 .
$$

As before, the measure $\mathcal{Q}$ satisfies (2.14) (with the constants $C_{n}, C^{\prime}, C^{\prime \prime}$, corresponding to $\left.v_{0}=0\right)$. Moreover, it is stationary in $\tau$.

Theorem 4.7. There exists a stationary solution $v(\tau)$ of the effective equation (2.25) such that $\mathcal{Q}=\mathcal{D}(I(v(\cdot)), V(v(\cdot)))$.

Proof. Denote $\mu^{\nu}=\mathcal{D} v^{\nu}(\tau)$. Estimate (1.5) with $2 m=r$ and $n=1$ implies that $\int|v|_{h^{r+1}}^{2} \mu^{\nu}(d v) \leq$ $C$ for all $\nu$. So the set of measures $\mu^{\nu}$ is tight in $\mathcal{H}^{r}$. Replacing, if necessary, the sequence $\left\{\nu_{l}\right\}$ by a subsequence, we achieve that

$$
\mu^{\nu_{l}} \rightarrow \mu^{0} \quad \text { as } \quad \nu_{l} \rightarrow 0 .
$$

Clearly $(I, V) \circ \mu^{0}$ is the marginal distribution for $\mathcal{Q}$ as $\tau=$ const, which we will denote $q$ (i.e., $\left.q=\left.\mathcal{Q}\right|_{\tau=\text { const }}\right)$.

Let $v^{0}(\tau), \tau \geq 0$, be a solution for the effective equation (2.25) such that $\mathcal{D} v^{0}(0)=\mu^{0}$ (existing by Corollary 4.3 and the estimates on $\mu^{0}$ ). Then, for the same reason as in Section 4.1,

$$
\left.\mathcal{D}(I, V)\left(v^{0}(\tau)\right)\right|_{\tau \in[0, T]}=\mathcal{Q},
$$

and $\mathcal{D}(I, V)\left(v^{0}(\tau)\right) \equiv q$. We do not know if the solution $v^{0}$ is stationary, but from the Bogolyubov-Krylov argument we know that for a suitable sequence $T_{j} \rightarrow \infty$ we have the convergence

$$
\frac{1}{T_{j}} \int_{0}^{T_{j}} \mathcal{D}\left(v^{0}(\tau)\right) d \tau \rightarrow m^{0},
$$

where $m^{0}$ is a stationary measure for $(2.25)$. Still we have that $(I, V) \circ m^{0}=q$, and the measure $m^{0}$ satisfies the same apriori estimates as before. Let $v(\tau)$ be a solution for $(2.25)$ such that $\mathcal{D} v(0)=m^{0}$. It is stationary and $\mathcal{D}(I, V)(v(\tau)) \equiv q$. Modifying a bit the argument above we get that also $\mathcal{D}(I, V)(v(\cdot))=\mathcal{Q}$.

Writing the convergence $(4.3)$ as $\mathcal{D}(I, V)\left(v^{\nu_{l}}(\cdot)\right) \rightarrow \mathcal{D}(I, V)(v(\cdot))$, we note that, as in Section 4.1 , we also have that

$$
\mathcal{D}\left(I, V^{\tilde{s}_{1}}, \ldots, V^{\tilde{s}_{m}}\right)\left(v^{\nu_{l}}(\tau)\right) \rightarrow \mathcal{D}\left(I, V^{\tilde{s}_{1}}, \ldots, V^{\tilde{s}_{m}}\right)(v(\tau))=\left(I, V^{\tilde{s}_{1}}, \ldots, V^{\tilde{s}_{m}}\right) \circ m^{0}
$$

as $\nu_{l} \rightarrow 0$, for any $m$ and any vectors $\tilde{s}_{1}, \ldots, \tilde{s}_{m}$, perpendicular to $\Lambda$. Since for stationary solutions $v^{\nu}(\tau)$ we have $\left\langle\mathcal{D}\left(v^{\nu}(\tau)\right)\right\rangle_{\tau_{1}}^{\tau_{2}}=\mathcal{D}\left(v^{\nu}(\tau)\right)$, then arguing as when proving Corollary 4.4 we also get that

$$
\mathcal{D}\left(I, \Phi^{\tilde{s}_{1}}, \ldots, \Phi^{\tilde{s}_{m}}\right)\left(v^{\nu_{l}}(\tau)\right) \rightarrow\left(I, \Phi^{\tilde{s}_{1}}, \ldots, \Phi^{\tilde{s}_{m}}\right) \circ m^{0} .
$$

\footnotetext{
${ }^{11}$ Under certain restrictions on the equation it is known that its law (i.e. the stationary measure of the equation) is unique, e.g., see [Shi06]. We will not discuss this now.
} 
Moreover if $s \in \mathbb{Z}_{0}^{\infty}$ is such that $s \cdot \Lambda \neq 0$, then in view of Proposition 4.10 and the stationarity of the solutions we have

$$
\mathcal{D}\left(I, \Phi^{\tilde{s}_{1}}, \ldots, \Phi^{\tilde{s}_{m}}, \Phi^{s}\right)\left(v^{\nu_{l}}(\tau)\right) \rightarrow\left(\left(I, \Phi^{\tilde{s}_{1}}, \ldots, \Phi^{\tilde{s}_{m}}\right) \circ m^{0}\right) \times d \theta .
$$

If eq. (2.25) has a unique stationary measure $m^{0}$, then the convergences above hold as $\nu \rightarrow 0$. But in this case a stronger assertion holds:

Theorem 4.8. Let $v^{\nu}$ be a stationary solution of equation $(2.1), \mathcal{D}\left(v^{\nu}(\tau)\right) \equiv \mu^{\nu}$, and assume that the effective equation (2.25) has a unique stationary measure $m^{0}$. Then

$$
\mu^{\nu} \rightarrow m^{0} \quad \text { as } \quad \nu \rightarrow 0 .
$$

Proof. i) Consider again the convergence (4.4). We are going to show that the limiting measure $\mu^{0}$ equals $m^{0}$. Then the limit in (4.4) does not depend on the sequence $\left\{\nu_{l} \rightarrow 0\right\}$, so it holds as $\nu \rightarrow 0$, and (4.7) follows.

ii) Due to Lemma $2.2, \mu^{\nu}(\partial)=0=\mu^{0}(\partial)$, so we may regard $\mu^{\nu}$ and $\mu^{0}$ as measures on $h_{I}^{r} \times \mathbb{T}^{\infty}$. Let us fix any $n \in \mathbb{N}$ and consider measures $\mu^{\nu n}, \mu^{0 n}$ and $m^{0 n}$ which are images of the measures $\mu^{\nu}, \mu^{0}$ and $m^{0}$ under the projection

$$
\Pi^{n}: v \mapsto v^{n}=\left(v_{1}, \ldots, v_{n}\right)
$$

(see Notation and Agreement). We will regard them as measures on $\mathbb{R}_{+}^{n} \times \mathbb{T}^{n}=\left\{\left(I^{n}, \varphi^{n}\right)\right\}$. To prove that $\mu^{0}=m^{0}$ it suffices to verify that $\mu^{0 n}=m^{0 n}$ for each $n$.

Let us denote $\mathcal{A}\left(\Lambda^{n}\right)=: \mathcal{A}^{n}$, and let the vectors $\zeta^{1}, \ldots, \zeta^{n} \in \mathbb{Z}^{n}$ and the unimodular matrix $R$ be as in Lemma 1.1 with $\mathcal{A}=\mathcal{A}^{n}$. Let $L=L_{\mathcal{A}^{n}}: \mathbb{T}^{n} \rightarrow \mathbb{T}^{n-1}$ be the operator in (1.9), i.e.

$$
L: \mathbb{T}^{n} \ni \varphi^{n} \mapsto\left(\varphi^{n} \cdot \zeta^{1}, \ldots, \varphi^{n} \cdot \zeta^{n-1}\right)^{T} \in \mathbb{T}^{n-1} .
$$

Writing $R^{T}\left(\varphi^{n}\right)=\left(y_{1}, \ldots, y_{n}\right)^{T}=\left(\mathbf{y}^{T}, y_{n}\right)^{T}$, where $\mathbf{y}=\left(y_{1}, \ldots, y_{n-1}\right)^{T}$, we have $L\left(\varphi^{n}\right)=\mathbf{y}$. We will denote by $\pi^{1}$ the natural projection $y \mapsto \mathbf{y}$.

For further purposes we make the following observation. Let $\mu$ be a Borel measure on $h$. Consider its images under rotations $\Psi_{t \Lambda}$ and projections $\Pi^{n}$. In the $(I, \varphi)$-variables the mapping $\Psi_{t \Lambda}$ becomes id $\times(\cdot+t \Lambda)$, so

$$
\Pi^{n} \circ\left(\Psi_{t \Lambda} \circ \mu\right)=\left(\mathrm{id} \times\left(\cdot+t \Lambda^{n}\right)\right) \circ \Pi^{n} \circ \mu
$$

(where $\Pi^{n} \circ \mu$ is written in the $\left(I^{n}, \varphi^{n}\right)$-variables). By (1.8) the transformation $R^{T}$ of $\mathbb{T}^{n}$ conjugates the translation by the vector $t \Lambda^{n}$ with the translation by $t e^{n}$. Therefore,

$$
\mathcal{R}^{T} \circ \Pi^{n} \circ\left(\Psi_{t \Lambda} \circ \mu\right)=\left(\mathrm{id} \times\left(\cdot+t e^{n}\right)\right) \circ \mathcal{R}^{T} \circ \Pi^{n} \circ \mu,
$$

where $\mathcal{R}^{T}=\mathrm{id} \times R^{T}$.

iii) Let us apply to the measures $\mu^{\nu n}, \mu^{0 n}, m^{0 n}$ the transformation $\mathcal{R}^{T}$ :

$$
N^{\nu n}=\mathcal{R}^{T} \circ \mu^{\nu n}, \quad N^{0 n}=\mathcal{R}^{T} \circ \mu^{0 n}, \quad M^{0 n}=\mathcal{R}^{T} \circ m^{0 n} .
$$

Recall that by (4.4), $N^{\nu_{l} n} \rightarrow N^{0 n}$ as $\nu_{l} \rightarrow 0$. Our first goal is to calculate the limiting measure $N^{0 n}$. To do this let us disintegrate $N^{\nu n}$ and $N^{0 n}$ with respect to the mapping

$$
\text { id } \times \pi^{1}: \mathbb{R}_{+}^{n} \times \mathbb{T}^{n} \rightarrow \mathbb{R}_{+}^{n} \times \mathbb{T}^{n-1}, \quad\left(I^{n},\left(\mathbf{y}^{T}, y_{n}\right)^{T}\right) \mapsto\left(I^{n}, \mathbf{y}\right) .
$$


That is (see [Dud02], Section 10.2), write them as

$$
N^{\nu n}=N_{I^{n}, \mathbf{y}}^{\nu n}\left(d y_{n}\right) p^{\nu n}\left(d I^{n} d \mathbf{y}\right), \quad N^{0 n}=N_{I^{n}, \mathbf{y}}^{0 n}\left(d y_{n}\right) p^{0 n}\left(d I^{n} d \mathbf{y}\right)
$$

where $p^{\nu n}=\left(\mathrm{id} \times \pi^{1}\right) \circ N^{\nu n}$ and $p^{0 n}=\left(\mathrm{id} \times \pi^{1}\right) \circ N^{0 n}$ Since $\mathbf{y}=L\left(\varphi^{n}\right)$, then $p^{\nu n}=\mathcal{D}\left(I^{n} \times\right.$ $\left.\left(L \circ \varphi^{n}\right)\right)\left(v^{\nu n}(\tau)\right)$. As each vector $\zeta^{j}$ in (4.8) is perpendicular to $\Lambda^{n}$, then in view of (4.5) we have

$$
p^{0 n}=\lim _{\nu_{l} \rightarrow 0} \mathcal{D}\left(I^{n} \times\left(L \circ \varphi^{n}\right)\right)\left(v^{\nu_{l} n}(\tau)\right)=\left(I^{n} \times\left(L \circ \varphi^{n}\right)\right) \circ m^{0 n} .
$$

To calculate $N^{0 n}$ it remains to find the fiber-measures $N_{I^{n}, \mathbf{y}}^{0}$. To do this let us take any bounded continuous function $f$ on $\mathbb{R}_{+}^{n} \times \mathbb{T}^{n-1} \times S^{1}$ and consider $\left\langle N^{\nu n}, f\right\rangle=\mathbf{E} f\left(I^{n}, \mathbf{y}, y_{n}\right)\left(v^{\nu}(\tau)\right)$. Since $\mathbf{y}(v)=L\left(\varphi^{n}\right)$ and $y_{n}(v)=v \cdot \eta^{n}$, where the vector $\eta^{n}$ is not perpendicular to $\Lambda$, then by (4.6)

$$
\left\langle N^{\nu n}, f\right\rangle \rightarrow \int f\left(I^{n}, \mathbf{y}, y_{n}\right)\left(\left(I^{n} \times\left(L \circ \varphi^{n}\right)\right) m^{0 n}\right)\left(d I^{n} d \mathbf{y}\right) d y_{n} .
$$

From other hand, by (4.4)

$$
\left\langle N^{\nu_{l} n}, f\right\rangle \rightarrow\left\langle N^{0 n}, f\right\rangle=\int f\left(I^{n}, \mathbf{y}, y_{n}\right) N_{I^{n}, \mathbf{y}}^{0 n}\left(d y_{n}\right) p^{0 n}\left(d I^{n} d \mathbf{y}\right)
$$

Since $p^{0 n}=\left(I^{n} \times\left(L \circ \varphi^{n}\right)\right) \circ m^{0 n}$, then we get from the two convergences above that for $p^{0 n}$-a.a. pairs $\left(I^{n}, \mathbf{y}\right)$ we have $N_{I^{n}, \mathbf{y}}^{0 n}=d y_{n}$. Accordingly,

$$
N^{0 n}=d y_{n} \times p^{0 n}\left(d I^{n} d \mathbf{y}\right) .
$$

iv) Consider the measure $M^{0}$. Due to (4.11) its disintegration with respect to the mapping id $\times \pi^{1}$ may be written as

$$
M^{0 n}=M_{I^{n}, \mathbf{y}}^{0 n}\left(d y_{n}\right) p^{0 n}\left(d I^{n} d \mathbf{y}\right)
$$

with some unknown fiber-measures $M_{I^{n}, \mathbf{y}}^{0}$. Now consider the rotated measure $\Psi_{t \Lambda} \circ m^{0}, t \geq 0$, and its $n$-dimensional projection. By (4.9),

$$
\mathcal{R}^{T} \circ \Pi^{n} \circ \Psi_{t \Lambda} \circ m^{0}=\left(\mathrm{id} \times l_{t}\right) \circ \mathcal{R}^{T} \circ m^{0 n},
$$

where $l_{t}\left(\mathbf{y}, y_{n}\right)=\left(\mathbf{y}, y_{n}+t\right)$. Due to (4.10) and (4.12), the measure in the r.h.s. equals

$$
M_{I^{n}, \mathbf{y}}^{0 n}\left(d y_{n}+t\right) p^{0 n}\left(d I^{n} d \mathbf{y}\right) .
$$

But by Corollary 2.10, the measure in the l.h.s. does not depend on $t$. So $M_{I^{n}, \mathbf{y}}^{0 n}\left(d y_{n}\right) \equiv$ $M_{I^{n}, \mathbf{y}}^{0 n}\left(d y_{n}+t\right)$ is a translation-invariant measure on $S^{1}$, and it must be equal to $d y_{n}$. Accordingly,

$$
M^{0 n}=đ y_{n} \times p^{0 n}\left(d I^{n} d y^{n}\right)=N^{0 n} .
$$

v) We have established that $N^{\nu_{l} n} \rightarrow M^{0 n}$ as $\nu_{l} \rightarrow 0$. So $\nu^{\nu_{l} n} \rightarrow m^{0 n}$, which completes the proof. 


\subsection{Mixing in the effective equations}

We start with the case when the function $f(\lambda)$ has a linear growth. For simplicity of notation we suppose that $f(\lambda)=\lambda+1$. We also are forced to assume that $q_{*}=1$.

The effective equation $(2.25)=(0.18)$ with $q_{*}=1$ looks similar to the equation $(0.6)_{\nu=\infty, q_{*}=1}$, studied in [KN13]. It turns out that the two equations indeed are similar, at least for $d \leq 3$, and that the proof of the mixing in Section 4 of [KN13], based on an abstract theorem from [KS12], applies to (2.25) with minimal changes. Indeed, the crucial step in [KN13] in order to apply the result from [KS12] is to establish for solutions of the equation the exponential estimate of the form

$$
\mathbf{P}\left\{\sup _{t \geq 0}\left(\int_{0}^{t}|u(s)|_{L_{\infty}}^{2} d s-K t\right) \geq \sigma\right\} \leq C^{\prime} \exp \left(c_{1}\left|u_{0}\right|_{L_{\infty}}^{2}-c_{2} \sigma\right), \quad \forall \sigma>0,
$$

with suitable constants $K, C^{\prime}, c_{1}$ and $c_{2}$. This estimate is important to study the mixing since it allows to control divergence of trajectories $u_{1}(t)$ and $u_{2}(t)$, corresponding to the same realisation of the random force, through the inequality ${ }^{12}$

$$
\left|u_{1}(t)-u_{2}(t)\right|_{L_{2}} \leq\left|u_{1}(0)-u_{2}(0)\right|_{L_{2}} \exp \left(C \int_{0}^{t}\left(\left|u_{1}(s)\right|_{L_{\infty}}^{2}+\left(\left|u_{2}(s)\right|_{L_{\infty}}^{2}\right) d s\right) .\right.
$$

For eq. (2.25) an analogy of (4.13) follows by applying the Ito formula to $[v]_{1}^{2}=H_{0}(v)+$ $H_{1}(v)$ (see $\left.(2.32)\right)$, since due to $(2.32)$ we have that

$$
d[v(\tau)]_{1}^{2}+2 \int_{0}^{\tau}[v(s)]_{2}^{2} d s=4 \tau \mathcal{B}+2 \sum_{j=1}^{\infty}\left(\lambda_{j}+1\right)\left(v_{j}(\tau) \cdot d \boldsymbol{\beta}^{j}(\tau)\right.
$$

where we denote $[v]_{2}^{2}=\sum\left(\lambda_{j}+1\right)^{2}\left|v_{j}\right|^{2}$ and $\mathcal{B}=\sum\left(\lambda_{j}+1\right) b_{j}^{2}$. Applying to this relation the supermartingale inequality in the standard way (e.g., see in [KN13, KS12]), we get that

$$
\mathbf{P}\left\{\sup _{\tau \geq 0}\left(\int_{0}^{\tau}[v(s)]_{2}^{2} d s-2 \mathcal{B} t\right) \geq \sigma\right\} \leq C^{\prime} \exp \left(c_{1}\left|v_{0}\right|_{1}^{2}-c_{2} \sigma\right), \quad \forall \sigma>0 .
$$

If $d \leq 3$, then by Lemma 2.11 the divergence of two solutions for $(2.25)$ with the same $\omega$ satisfies

$$
\left|v_{1}(\tau)-v_{2}(\tau)\right|_{h^{0}} \leq\left|v_{1}(0)-v_{2}(0)\right|_{h^{0}} \exp \left(C \int_{0}^{\tau}\left(\left[v_{1}(s)\right]_{2}^{2}+\left[v_{2}(s)\right]_{2}^{2}\right) d s\right) .
$$

This last two estimates allow to repeat literally for equation (2.25) the reduction to Theorem 3.1.3 from [KS12], made in [KN13], and prove

Theorem 4.9. Let $q_{*}=1, f(\lambda)=\lambda+1$ and $d \leq 3$. Then the effective equation (2.25) has a unique stationary measure $\mu$ and is mixing. That is, every its solution $v(\tau)$ satisfies $\mathcal{D}(v(\tau)) \rightarrow \mu$ as $\tau \rightarrow \infty$.

The presented proof uses that the nonlinearity in the effective equation is at most cubic. It also applies to the effective equations for eq. (0.6), where the Hamiltonian $\mathcal{H}$ is one of the

\footnotetext{
${ }^{12}$ To match (4.13) and (4.14) we use crucially that $q_{*} \leq 1$.
} 
two functions $\mathcal{H}^{3}$ with cubic densities as at the end of Section 0.1 (in this case the argument works if $d \leq 6)$. The proof without changes applies to equation $(0.6)$, where $q_{*}=1, d \leq 3$ and $f(\lambda)$ grows super-linearly. The argument also may be adjusted to the case when $q_{*}=1, d$ is any and $f(\lambda)=c_{1}+\lambda^{c_{d}}$, where $c_{d}$ is sufficiently big. Based on the similarity with the equation $(0.6)_{\nu=\infty, q_{*}=1}$, studied in [KN13] for any space-dimension, we conjecture that for $q_{*}=1$ and $f(\lambda)=\lambda+1$ the effective equation is well-posed and mixing for any $d$. But it is unknown how to prove the mixing for equations with $q_{*} \geq 2$ (in any space-dimension).

\subsection{Proof of Lemma 4.6}

For this proof we adopt a notation from [KP08]. Namely, we denote by $\varkappa(t)$ various functions of $t$ such that $\varkappa \rightarrow 0$ as $t \rightarrow \infty$, and denote by $\varkappa_{\infty}(t)$ functions, satisfying $\varkappa(t)=o\left(t^{-N}\right)$ for each $N$. We write $\varkappa(t, M)$ to indicate that $\varkappa(t)$ depends on a parameter $M$. Besides for events $Q$ and $O$ and a random variable $f$ we write $\mathbf{P}_{O}(Q)=\mathbf{P}(O \cap Q)$ and $\mathbf{E}_{O}(f)=\mathbf{E}\left(\chi_{O} f\right)$. Below $M$ stands for a suitable function of $\nu$ such that $M(\nu) \rightarrow \infty$ as $\nu \rightarrow 0$, but

$$
\nu M^{n} \rightarrow 0 \quad \text { as } \nu \rightarrow 0, \quad \forall n
$$

Denote by $\Omega_{M}=\Omega_{M}^{\nu}$ the event $\Omega_{M}=\left\{\sup _{0 \leq \tau \leq T}\left|a^{\nu}(\tau)\right|_{h^{r}} \leq M\right\}$. Then, by (1.6), $\mathbf{P}\left(\Omega_{M}^{c}\right) \leq \varkappa_{\infty}(M)$ uniformly in $\nu$, so that one has $\mathfrak{A}_{k}^{\nu} \leq \varkappa_{\infty}(M)+\mathfrak{A}_{k, M}^{\nu}$, where we have defined

$$
\mathfrak{A}_{k, M}^{\nu}:=\mathbf{E}_{\Omega_{M}} \max _{0 \leq \tau \leq T}\left|\int_{0}^{\tau} \mathcal{R}_{k}\left(a^{\nu}(s), \nu^{-1} s\right) d s\right| .
$$

So it remains to estimate $A_{k, M}^{\nu}$.

Consider a partition of $[0, T]$ by the points

$$
\tau_{n}=n L, \quad 0 \leq n \leq K \sim T / L .
$$

where $\tau_{K}$ is the last point $\tau_{n}$ in $[0, T)$. The diameter $L$ of the partition is $L=\sqrt{\nu}$. Denoting

$$
\eta_{l}=\int_{\tau_{l}}^{\tau_{l+1}} \mathcal{R}_{k}\left(a^{\nu}(s), \nu^{-1} s\right) d s, \quad 0 \leq l \leq K-1
$$

we see that

$$
\mathfrak{A}_{k, M}^{\nu} \leq L C(M)+\mathbf{E}_{\Omega_{M}} \sum_{l=0}^{K-1}\left|\eta_{l}\right|
$$

since for $\omega \in \Omega_{M}$ the integrand in (4.16) is smaller than a suitable $C(M)$ (see Lemma 2.1 and $(2.21))$. For any $l$ let us consider the event

$$
\mathcal{F}_{l}=\left\{\sup _{\tau_{l} \leq \tau \leq \tau_{l+1}}\left|a^{\nu}(\tau)-a^{\nu}\left(\tau_{l}\right)\right|_{h} \geq P_{1}(M) L^{1 / 3}\right\}
$$

where $P_{1}(M)$ is a suitable polynomial. It is not hard to verify using the Doob inequality that for a suitable choice of $P_{1}$ the probability of $\mathbf{P}\left(\mathcal{F}_{l}\right)$ is less than $\varkappa_{\infty}\left(L^{-1} ; M\right)$ (cf. [KP08]). One gets

$$
\sum_{l=0}^{K-1}\left|\mathbf{E}_{\Omega_{M}}\right| \eta_{l}\left|-\mathbf{E}_{\Omega_{M} \backslash \mathcal{F}_{l}}\right| \eta_{l}|| \leq C(M) L \sum_{l=0}^{K-1} \mathbf{P}\left(\mathcal{F}_{l}\right) \leq C(M) \varkappa_{\infty}\left(L^{-1} ; M\right)
$$


so that it remains to estimate $\sum \mathbf{E}_{\Omega_{M} \backslash \mathcal{F}_{l}}\left|\eta_{l}\right|$.

We have

$$
\begin{aligned}
\left|\eta_{l}\right| & \leq\left|\int_{\tau_{l}}^{\tau_{l+1}}\left(\mathcal{R}_{k}\left(a^{\nu}(s), \nu^{-1} s\right)-\mathcal{R}_{k}\left(a^{\nu}\left(\tau_{l}\right), \nu^{-1} s\right)\right) d s\right| \\
& +\left|\int_{\tau_{l}}^{\tau_{l+1}}\left(\mathcal{R}_{k}\left(a^{\nu}\left(\tau_{l}\right), \nu^{-1} s\right)\right) d s\right|=: \Upsilon_{l}^{1}+\Upsilon_{l}^{2} .
\end{aligned}
$$

By the regularity of the integrand and the definition of $\mathcal{F}_{l}$

$$
\sum_{l} \mathbf{E}_{\Omega_{M} \backslash \mathcal{F}_{l}} \Upsilon_{l}^{1} \leq \varkappa\left(L^{-1 / 3} ; M\right)=\varkappa\left(\nu^{-1 / 6} ; M\right) .
$$

So it remains to estimate the expectation of $\sum \Upsilon_{l}^{2}$. Denoting $t=\nu \tau$ and making use of (2.29) we write $\Upsilon_{l}^{2}$ as

$$
\begin{aligned}
\Upsilon_{l}^{2} & =L\left|\frac{\nu}{L} \int_{0}^{\nu^{-1} L} \sum_{\substack{p, q, l \in \mathbb{Z}_{+0}^{\infty} \\
q-l-e^{k} \notin \mathcal{A}(\Lambda, m) \\
|q|+|l|+1 \leq m}} P_{k}^{0 p q l}(a) \exp \left(-i t\left(\Lambda \cdot\left(q-l-e^{k}\right)\right)\right) d t\right| \\
& \leq L C(M) \frac{\nu}{L} \sup _{\substack{p, q, l \in \mathbb{Z}_{+0}^{\infty} \\
q-l-e^{k} \notin \mathcal{A}(\Lambda, m) \\
|q|+|l|+1 \leq m}} \frac{1}{\Lambda \cdot\left(q-l-e^{k}\right)} \leq L \varkappa\left(\nu^{-1} L ; M\right),
\end{aligned}
$$

because the supremum in the second line is bounded by one, since both $\Lambda$ and $q-l-e^{k}$ are integer vectors. Therefore

$$
\sum_{l} \mathbf{E}_{\Omega_{M} \backslash \mathcal{F}_{l}} \Upsilon_{l}^{2} \leq \varkappa\left(\nu^{-1 / 2} ; M\right) .
$$

Now (4.15), (4.17), (4.18), (4.19) and (4.20) imply that

$$
\mathfrak{A}_{k}^{\nu} \leq \varkappa_{\infty}(M)+\varkappa\left(\nu^{-1 / 2} ; M\right)+\varkappa_{\infty}\left(\nu^{-1} ; M\right)+\varkappa\left(\nu^{-1 / 6} ; M\right)+\varkappa\left(\nu^{-1 / 2} ; M\right) .
$$

Choosing first $M$ large and then $\nu$ small, we make the r.h.s. above arbitrarily small. This proves the lemma.

An argument similar to the previous one (see Appendix A) implies the following assertion:

Proposition 4.10. Let $s \in \mathbb{Z}_{0}^{\infty}$ be such that $s \cdot \Lambda \neq 0$ and $G: \mathbb{R}_{+}^{M} \times \mathbb{T}^{J(M)} \times S^{1} \rightarrow \mathbb{R}$ be a bounded Lipschitz-continuous function, for some $M \geq 1$. Then

$$
\begin{aligned}
\mathfrak{B}^{\nu}:=\mathbf{E} \max _{0 \leq \tau \leq T} \mid & \int_{0}^{\tau}\left(G\left(I^{\nu M}(l), \Phi^{\nu(M)}(l), s \cdot \varphi^{\nu}(l)\right)-\right. \\
& \left.\int_{S^{1}} G\left(I^{\nu M}(l), \Phi^{\nu(M)}(l), \theta\right) d \theta\right) d l \mid \rightarrow 0 \quad \text { as } \quad \nu \rightarrow 0 .
\end{aligned}
$$

In particular, taking for $G$ Lipschitz functions on $S^{1}$ we get that $\left\langle\mathcal{D}\left(s \cdot \varphi^{\nu}(l)\right)\right\rangle_{0}^{t} \rightarrow d \theta$ as $\nu \rightarrow 0$, for any $t>0$. 


\section{A Proof of Proposition 4.10}

For this proof, as in Section 4.4, we denote by $\varkappa(t)$ various functions of $t$ such that $\varkappa \rightarrow 0$ as $t \rightarrow \infty$, and denote by $\varkappa_{\infty}(t)$ functions, satisfying $\varkappa(t)=o\left(t^{-N}\right)$ for each $N$. For events $Q$ and $\mathcal{O}$ and a random variable $f$ we write $\mathbf{P}_{\mathcal{O}}(Q)=\mathbf{P}(\mathcal{O} \cap Q)$ and $\mathbf{E}_{\mathcal{O}}(f)=\mathbf{E}\left(\chi_{\mathcal{O}} f\right)$. Without lost of generality we assume that $|G| \leq 1$ and $\operatorname{Lip} G \leq 1$.

Let us denote by $R$ a suitable function of $\nu$ such that $R(\nu) \rightarrow \infty$ as $\nu \rightarrow 0$, but

$$
\nu R^{n} \rightarrow 0 \quad \text { as } \nu \rightarrow 0, \quad \forall n .
$$

Denote, moreover, by $\Omega_{R}=\Omega_{R}^{\nu}$ the event $\Omega_{R}=\left\{\sup _{0 \leq \tau \leq T}\left|v^{\nu}(\tau)\right|_{r} \leq R\right\}$. Then, by (1.6), $\mathbf{P}\left(\Omega_{R}^{c}\right) \leq \varkappa_{\infty}(R)$ uniformly in $\nu$.

Taking into account the boundedness of $G$, we get that

$$
\begin{aligned}
\mathfrak{B}^{\nu} \leq \varkappa_{\infty}(R)+\mathbf{E}_{\Omega_{R}} \max _{0 \leq \tau \leq T} \mid & \int_{0}^{\tau}\left(G\left(I^{\nu M}(l), \Phi^{\nu(M)}(l), s \cdot \varphi^{\nu}(l)\right)\right. \\
& \left.-\int_{S^{1}} G\left(I^{\nu M}(l), \Phi^{\nu(M)}(l), \theta\right) d \theta\right) d l \mid .
\end{aligned}
$$

As in the proof of Lemma 4.6, consider a partition of $[0, T]$ by the points

$$
\tau_{n}=\tau_{0}+n L, \quad 0 \leq n \leq K \sim T / L .
$$

where $\tau_{K}$ is the last point $\tau_{n}$ in $[0, T)$. The diameter $L$ of the partition is $L=\sqrt{\nu}$, and the non-random phase $\tau_{0} \in[0, L)$ will be chosen later. Denoting

$$
\eta_{n}=\int_{\tau_{n}}^{\tau_{n+1}}\left(G\left(I^{\nu M}, \Phi^{\nu(M)}, s \cdot \varphi^{\nu}\right)-\int_{S^{1}} G\left(I^{\nu M}, \Phi^{\nu(M)}, \theta\right) d \theta\right) d l, \quad 0 \leq n \leq K-1,
$$

we see that

$$
\mathfrak{B}^{\nu} \leq \varkappa_{\infty}(R)+C L+\mathbf{E}_{\Omega_{R}} \sum_{n=0}^{K-1}\left|\eta_{n}\right|,
$$

so it remains to estimate $\sum \mathbf{E}_{\Omega_{R}}\left|\eta_{n}\right|$. We abbreviate

$$
\hat{G}(\psi ; l)=G\left(I^{\nu M}(l), \Phi^{\nu(M)}(l), \psi\right), \quad \psi \in S^{1},
$$

so that we have

$$
\begin{aligned}
\left|\eta_{n}\right| & \left.\leq \mid \int_{\tau_{n}}^{\tau_{n+1}}\left(\hat{G}\left(s \cdot \varphi^{\nu}(l) ; l\right)\right)-\hat{G}\left(s \cdot \varphi^{\nu}\left(\tau_{n}\right)+\nu^{-1}(s \cdot \Lambda)\left(l-\tau_{n}\right) ; \tau_{n}\right)\right) d l \mid \\
& +\left|\int_{\tau_{n}}^{\tau_{n+1}}\left(\hat{G}\left(s \cdot \varphi^{\nu}\left(\tau_{n}\right)+\nu^{-1}(s \cdot \Lambda)\left(l-\tau_{n}\right) ; \tau_{n}\right)-\int_{S^{1}} \hat{G}\left(\theta ; \tau_{n}\right) d \theta\right) d l\right| \\
& +\left|\int_{\tau_{l}}^{\tau_{l+1}}\left(\int_{S^{1}} \hat{G}\left(\theta ; \tau_{n}\right) d \theta-\int_{S^{1}} \hat{G}(\theta ; l) d \theta\right) d l\right|=: \Upsilon_{n}^{1}+\Upsilon_{n}^{2}+\Upsilon_{n}^{3} .
\end{aligned}
$$

To estimate the quantities $\Upsilon_{n}^{1,2,3}$ we first optimise the choice of the phase $\tau_{0}$. A crucial point here is that, if we set $N:=M \vee\lceil s\rceil$, the function $G$ depends only on $v^{N}$. So we consider the events $\mathcal{E}_{n}, 1 \leq n \leq K$,

$$
\mathcal{E}_{n}=\left\{I_{k}^{\nu}\left(\tau_{n}\right) \leq \varepsilon \text { for some } k \leq N\right\}, \quad \text { where } \varepsilon \geq \nu^{a}, \quad a=1 / 10 .
$$


Since for each $k \leq M$ by Lemma 2.2 we have

$$
\int_{0}^{L} \sum_{n=0}^{K} \mathbf{P}\left(I_{k}^{\nu}\left(\bar{\tau}_{n}\right) \leq \varepsilon\right) d \bar{\tau}_{0}=\int_{0}^{T} \mathbf{P}\left(I_{k}^{\nu}(\tau) \leq \varepsilon\right) d \tau=\varkappa\left(\varepsilon^{-1} ; R, N\right)
$$

(here each $\bar{\tau}_{n}$ is regarded as a function of $\tau_{0}=\bar{\tau}_{0}$, given by (A.1)), then we can choose $\tau_{0} \in[0, L)$ in such a way that

$$
K^{-1} \sum_{n=0}^{K-1} \mathbf{P}\left(\mathcal{E}_{n}\right)=\varkappa\left(\varepsilon^{-1} ; R, N\right)
$$

For any $n$ consider the event

$$
\mathcal{Q}_{n}=\left\{\sup _{\tau_{n} \leq \tau \leq \tau_{n+1}}\left|I^{\nu}(\tau)-I^{\nu}\left(\tau_{n}\right)\right|_{h_{I}} \geq P_{1}(R) L^{1 / 3}\right\},
$$

where $P_{1}(R)$ is a suitable polynomial. It is not hard to verify using the Doob inequality that its probability satisfies $\mathbf{P}\left(\mathcal{Q}_{n}\right) \leq \varkappa_{\infty}\left(L^{-1}\right)$ (cf. [KP08]). Setting $\mathcal{F}_{n}=\mathcal{E}_{n} \cup \mathcal{Q}_{n}, n=0, \ldots, K-1$, we have that

$$
\frac{1}{K} \sum_{n=0}^{K-1} \mathbf{P}\left(\mathcal{F}_{n}\right) \leq \varkappa\left(\varepsilon^{-1} ; R, N\right)+\varkappa_{\infty}\left(\nu^{-1 / 2} ; N\right)=: \tilde{\varkappa} .
$$

Accordingly,

$$
\sum_{n=0}^{K-1}\left|\left(\mathbf{E}_{\mathcal{F}_{n} \cap \Omega_{R}}\right) \Upsilon_{n}^{j}\right| \leq C L \sum_{n=0}^{K-1} \mathbf{P}\left(\mathcal{F}_{n}\right) \leq C \tilde{\varkappa}:=\tilde{\varkappa}_{1}, \quad j=1,2,3
$$

If $\omega \in \Omega_{R} \backslash \mathcal{F}_{n}$, then for $\tau \in\left[\tau_{n}, \tau_{n+1}\right]$ we have that $I_{k}^{\nu}(\tau) \geq \varepsilon-P_{1}(R) L^{1 / 3} \geq \frac{1}{2} \varepsilon$. On the other hand, by Lemma 2.1, for any positive $\delta$ we have the estimate

$$
\left.|| v_{k}\right|^{-2}\left(i v_{k} \cdot P_{k}(v)\right) \chi_{\left\{\left|v_{k}\right|>\delta\right\}} \mid \leq \delta^{-1} Q_{k}\left(|v|_{h^{r}}\right),
$$

where $Q_{k}$ is a polynomial. These relations and (2.6) imply that

$$
\begin{aligned}
\mathbf{P}_{\Omega_{R} \backslash \mathcal{F}_{n}}\left\{\left|\varphi^{\nu N}(l)-\left(\varphi^{\nu M}\left(\tau_{n}\right)+\nu^{-1} \Lambda^{N}\left(l-\tau_{n}\right)\right)\right| \geq \nu^{a}\right. \text { for some } & \left.l \in\left[\tau_{n}, \tau_{n+1}\right]\right\} \\
& \leq \varkappa_{\infty}\left(\nu^{-1} ; R, N\right)
\end{aligned}
$$

(cf. the estimate of $\mathbf{P}\left(\mathcal{Q}_{n}\right)$ ). Therefore

$$
\begin{aligned}
& \mathbf{P}_{\Omega_{R} \backslash \mathcal{F}_{n}}\left\{\left|s \cdot \varphi^{\nu N}(l)-\left(s \cdot \varphi^{\nu N}\left(\tau_{n}\right)+\nu^{-1}(s \cdot \Lambda)\left(l-\tau_{n}\right)\right)\right|\right. \\
& \left.\quad \text { for some } l \in\left[\nu_{n}^{a}, \tau_{n+1}\right]\right\} \leq \varkappa_{\infty}\left(\nu^{-1} ; R, N, s, \Lambda\right) .
\end{aligned}
$$

and

$$
\mathbf{P}_{\Omega_{R} \backslash \mathcal{F}_{n}}\left\{\left|\Phi^{\nu(M)}(l)-\Phi^{\nu(M)}\left(\tau_{n}\right)\right| \geq \nu^{a} \text { for some } l \in\left[\tau_{n}, \tau_{n+1}\right]\right\} \leq \varkappa_{\infty}\left(\nu^{-1} ; R, N\right) .
$$

From here and the definition of the events $\mathcal{Q}_{n}$ we find that

$$
\sum_{l} \mathbf{E}_{\Omega_{R} \backslash \mathcal{F}_{n}} \Upsilon_{n}^{1} \leq C(R) \nu^{1 / 6}+C(R) \nu^{a}+\varkappa_{\infty}\left(\nu^{-1} ; R, N, s \cdot \Lambda\right) .
$$


For the same reason also

$$
\sum_{l} \mathbf{E}_{\Omega_{R} \backslash \mathcal{F}_{n}} \Upsilon_{n}^{3} \leq C(R) \nu^{1 / 6}+C(R) \nu^{a}+\varkappa_{\infty}\left(\nu^{-1} ; R, N, s \cdot \Lambda\right)
$$

So it remains to estimate the expectation of $\sum \Upsilon_{n}^{2}$. Denoting $t=\nu\left(l-\tau_{n}\right)$ we write $\Upsilon_{n}^{2}$ as

$$
\begin{aligned}
\Upsilon_{n}^{2} & =\left|\int_{\tau_{n}}^{\tau_{n+1}} \hat{G}\left(s \cdot \varphi^{\nu}\left(\tau_{n}\right)+\nu^{-1}(s \cdot \Lambda)\left(l-\tau_{n}\right) ; \tau_{n}\right) d l-L \int_{S^{1}} \hat{G}\left(\theta ; \tau_{n}\right) d \theta\right| \\
& =L\left|\frac{\nu}{L} \int_{0}^{\nu^{-1} L} \hat{G}\left(s \cdot \varphi^{\nu}\left(\tau_{n}\right)+s \cdot \Lambda t ; \tau_{n}\right) d t-\int_{S^{1}} \hat{G}\left(\theta ; \tau_{n}\right) d \theta\right| .
\end{aligned}
$$

Let us expand $\hat{G}\left(\psi ; \tau_{n}\right)$ as a Fourier series $\hat{G}(\psi)=\sum g_{k} e^{i k \psi}$, where each $g_{k}$ is a random variable and $g_{0}=\int_{S^{1}} \hat{G}\left(\theta ; \tau_{n}\right) d \theta$ (we discard the dependence on $\tau_{n}$, which is fixed thought the argument). Then

$$
\left|\frac{1}{T} \int_{0}^{T} \hat{G}\left(\psi_{0}+t(s \cdot \Lambda)\right) d t-g_{0}\right| \leq \varepsilon \quad \forall T \geq T_{\varepsilon},
$$

for a suitable non-random $T_{\varepsilon}$. Indeed, for each nonzero $k$, one has

$$
\left|\frac{1}{T} \int_{0}^{T} e^{i k\left(\psi_{0}+t(s \cdot \Lambda)\right)} d t\right| \leq \frac{2}{T|s \cdot \Lambda|},
$$

so that ${ }^{13}$

$$
\left|\frac{1}{T} \int_{0}^{T} \hat{G}\left(\psi_{0}+t(s \cdot \Lambda)\right) d t-g_{0}\right| \leq \frac{2}{T|s \cdot \Lambda|} \sum\left|g_{k}\right| \leq \frac{2 C}{T|s \cdot \Lambda|} .
$$

We have thus proved that $\Upsilon_{n}^{2} \leq L \varkappa\left(\nu^{-1} L ; R, N, \varepsilon, s \cdot \Lambda\right)$. Therefore

$$
\sum_{l} \mathbf{E}_{\Omega_{R} \backslash \mathcal{F}_{l}^{c}} \Upsilon_{n}^{2} \leq \varkappa\left(\nu^{-1 / 2} ; R, N, \varepsilon, s \cdot \Lambda\right) .
$$

Now (A.3), (A.5) and (A.6)-(A.8) imply that $\mathfrak{B}^{\nu}$ is bounded by

$$
\varkappa_{\infty}(R)+\varkappa\left(\nu^{-a} ; R, N\right)+\varkappa\left(\varepsilon^{-1} ; R, N\right)+C(R) \nu^{a}+C(R) \nu^{1 / 6}+\varkappa\left(\nu^{-1 / 2} ; R, N, \varepsilon, s \cdot \Lambda\right) .
$$

Choosing first $R$ large and next $\varepsilon$ small and $\nu$ small in such a way that (A.4) holds, we make the quantity above arbitrarily small. This proves the required convergence.

The second assertion of the proposition follows from the first one, since to check the weak convergence of measures on a complete metric space it suffices to take for test-functions the Lipschitz functions.

\footnotetext{
${ }^{13}$ By the Bernstein theorem, $\sum_{k=1}^{\infty}\left|g_{k}\right| \leq C$, where the constant $C=C(\hat{G})$ is finite if the function $\hat{G}(\psi)$ is Lipschitz-continuous. The proof of the theorem (e.g., see [Zyg59], Section VI.3) easily implies that $C$ depends only on the Lipschitz constant of $\hat{G}$, which equals 1 in our case.
} 


\section{References}

[AKN06] V. Arnold, V. V. Kozlov, and A. I. Neistadt, Mathematical Aspects of Classical and Celestial Mechanics, third ed., Springer, Berlin, 2006.

[Bou71] N. Bourbaki, Éléments de Mathématique. Livre III: Topologie Génerale, Chap. 5 à 10, Hermann, Paris, 1971.

[CFG08] J. Cardy, G. Falkovich, and K. Gawedzki, Non-equilibrium Statistical Mechanics and Turbulence, Cambridge University Press, Cambridge, 2008.

[Dud02] R. M. Dudley, Real Analysis and Probability, Cambridge University Press, Cambridge, 2002.

[FGH15] E. Faou, P. Germain, and Z. Hani, The weakly nonlinear large box limit of the 2D cubic nonlinear Schrödinger equation, Journal of the American Mathematical Society (2015), in press.

[FW03] M. I. Freidlin and A. D. Wentzell, Averaging principle for stochastic perturbations of multifrequency systems, Stochastics and Dynamics 3 (2003), 393-408.

[GG12] P. Gérard and S. Grellier, Effective integrable dynamics for a certain nonlinear wave eqaution, Analysis and PDE 5 (2012), 1139-1154.

[Hai02] M. Hairer, Exponential mixing properties of stochastic PDE's through asymptotic coupling, Probab. Theory Relat. Fields 124 (2002), 345-380.

[Kar10] E. Kartashova, Nonlinear resonance analysis: theory, computation, applications, Cambridge University Press, Cambridge, 2010.

[Kha68] R. Khasminski, On the avaraging principle for Ito stochastic differential equations, Kybernetika 4 (1968), 260-279, (in Russian).

[KM15a] S. Kuksin and A. Maiocchi, Derivation of a wave kinetic equation from the resonantaveraged stochastic NLS equation, Physica D 309 (2015), 65-70.

[KM15b] _ The limit of small Rossby numbers for randomly forced quasi-geostrophic equation on $\beta$-plane, Nonlinearity 28 (2015), 2319-3241.

[KN13] S. Kuksin and V. Nersesyan, Stochastic CGL equations without linear dispersion in any space dimension, Stoch PDE: Anal Comp 1 (2013), 389-423.

[KP08] S. B. Kuksin and A. L. Piatnitski, Khasminskii - Whitham averaging for randomly perturbed KdV equation, J. Math. Pures Appl. 89 (2008), 400-428.

[KS91] I. Karatzas and S. Shreve, Brownian Motion and Stochastic Calculus, 2nd ed., Springer-Verlag, Berlin, 1991.

[KS04] S. B. Kuksin and A. Shirikyan, Randomly forced CGL equation: stationary measures and the inviscid limit, J. Phys. A: Math. Gen. 37 (2004), 1-18. 
[KS12] S. Kuksin and A. Shirikyan, Mathematics of Two-Dimensional Turbulence, Cambridge University Press, Cambridge, 2012.

[Kuk10] S. B. Kuksin, Damped-driven KdV and effective equations for long-time behaviour of its solutions, GAFA 20 (2010), 1431-1463.

[Kuk13]_ Weakly nonlinear stochastic CGL equations, Ann. Inst. H. Poincaré - PR 49 (2013), 1033-1056.

[MR99] R. Mikulevicius and B. L. Rozovskii, Martingale problems for stochastic PDEs, Stochastic partial differential equations: six perspectives, Math. Surveys Monogr., no. 64, Amer. Math. Soc., Providence, RI, 1999, pp. 243-325.

[Naz11] S. Nazarenko, Wave Turbulence, Springer, Berlin, 2011.

[Oda06] C. Odasso, Ergodicity for the stochastic complex Ginzburg-Landau equations, Ann. Inst. H. Poincaré - PR 42 (2006), 417-454.

[Pei97] R. Peierls, On the kinetic theory of thermal conduction in crystals, Selected Scientific Papers of Sir Rudolf Peierls, with commentary, World Scientific, Singapore, 1997, pp. 15-48.

[Shi06] A. Shirikyan, Ergodicity for a class of Markov processes and applications to randomly forced PDE's. II, DCDS-A 6 (2006), 911-926.

[VF88] M. I. Vishik and A. V. Fursikov, Mathematical Problems in Statistical Hydromechanics, Kluwer, Dordrecht, 1988.

[Yor74] M. Yor, Existence et unicité de diffusion à valeurs dans un espace de Hilbert, Ann. Inst. Henri Poincaré Sec. B, 10 (1974), 55-88.

[ZL75] V. E. Zakharov and V. S. L'vov, Statistical description of nonlinear wave fields, Radiophys. Quan. Electronics 18 (1975), 1084-1097.

[ZLF92] V. Zakharov, V. L'vov, and G. Falkovich, Kolmogorov Spectra of Turbulence, Springer, Berlin, 1992.

[Zyg59] A. Zygmund, Trigonometric Series, vol. 1, Cambridge University Press, Cambridge, 1959. 